REPRESENTATION THEORY

An Electronic Journal of the American Mathematical Society

Volume 12, Pages 236-259 (July 29, 2008)

S 1088-4165(08)00333-6

\title{
CENTERS OF DEGENERATE CYCLOTOMIC HECKE ALGEBRAS AND PARABOLIC CATEGORY $O$
}

\author{
JONATHAN BRUNDAN
}

\begin{abstract}
We prove that the center of each degenerate cyclotomic Hecke algebra associated to the complex reflection group of type $B_{d}(l)$ consists of symmetric polynomials in its commuting generators. The classification of the blocks of the degenerate cyclotomic Hecke algebras is an easy consequence. We then apply Schur-Weyl duality for higher levels to deduce analogous results for parabolic category $\mathcal{O}$ for the Lie algebra $\mathfrak{g l}_{n}(\mathbb{C})$.
\end{abstract}

\section{INTRODUCTION}

Let $R$ be a fixed commutative ground ring. Recall from $\mathrm{D}$ that the degenerate affine Hecke algebra $H_{d}$ is the $R$-algebra which is equal as an $R$-module to the tensor product $R\left[x_{1}, \ldots, x_{d}\right] \otimes_{R} R S_{d}$ of the polynomial algebra $R\left[x_{1}, \ldots, x_{d}\right]$ and the group algebra $R S_{d}$ of the symmetric group $S_{d}$. Multiplication is defined so that $R\left[x_{1}, \ldots, x_{d}\right]$ (identified with the subspace $R\left[x_{1}, \ldots, x_{d}\right] \otimes 1$ ) and $R S_{d}$ (identified with the subspace $1 \otimes R S_{d}$ ) are subalgebras, and in addition

$$
\begin{aligned}
s_{i} x_{i+1} & =x_{i} s_{i}+1, \\
s_{i} x_{j} & =x_{j} s_{i} \quad(j \neq i, i+1),
\end{aligned}
$$

where $s_{i}$ denotes the basic transposition $(i i+1) \in S_{d}$. It is known by $\mathrm{L}$, Theorem 6.5] that the center $Z\left(H_{d}\right)$ of $H_{d}$ consists of all symmetric polynomials in the (algebraically independent) generators $x_{1}, \ldots, x_{d}$.

Given in addition a monic polynomial $f(x)=x^{l}+c_{1} x^{l-1}+\cdots+c_{l} \in R[x]$ of degree $l \geq 1$, the degenerate cyclotomic Hecke algebra $H_{d}^{f}$ is the quotient of $H_{d}$ by the two-sided ideal generated by $f\left(x_{1}\right)$. We refer to $l$ here as the level. Since we seldom mention $H_{d}$ itself again, it should not cause confusion to also use the notation $x_{1}, \ldots, x_{d}$ for the canonical images of the polynomial generators of $H_{d}$ in the quotient $H_{d}^{f}$. For example, if $f(x)=x$, then $H_{d}^{f}$ can be identified simply with the group algebra $R S_{d}$, and under this identification we have that

$$
x_{i}=\sum_{j=1}^{i-1}(j i) \in R S_{d}
$$

the Jucys-Murphy elements. In this case, it has long been known (see $\mathrm{Ju}$ or [M, 1.9]) that the center of $R S_{d}$ again consists of all symmetric polynomials in

Received by the editors August 15, 2006 and, in revised form, June 25, 2008.

2000 Mathematics Subject Classification. Primary 20C08, 17B20.

Research supported in part by NSF grant no. DMS-0654147.

(C)2008 American Mathematical Society 
$x_{1}, \ldots, x_{d}$, though of course these generators are no longer algebraically independent. In other words, the canonical homomorphism $H_{d} \rightarrow R S_{d}$ maps $Z\left(H_{d}\right)$ surjectively onto $Z\left(R S_{d}\right)$. Our first result proves the analogous statement for the quotient map $H_{d} \rightarrow H_{d}^{f}$ in general.

Theorem 1. The center of $H_{d}^{f}$ consists of all symmetric polynomials in $x_{1}, \ldots, x_{d}$. Moreover, $Z\left(H_{d}^{f}\right)$ is free as an $R$-module with an explicit basis parametrized by all l-multipartitions of $d$.

For the first application, specialize to the case that $R=F$ is an algebraically closed field. We say that two irreducible modules $L$ and $L^{\prime}$ belong to the same block if they are linked by a chain $L=L_{0}, L_{1}, \ldots, L_{n}=L^{\prime}$ of irreducible modules such that there is a non-split extension between $L_{i-1}$ and $L_{i}$ for each $i=1, \ldots, n$. For modules over a finite dimensional algebra like $H_{d}^{f}$, this is equivalent to the property that $L$ and $L^{\prime}$ have the same central character. So, on combining Theorem 1 with the existing theory, we obtain the classification of the blocks of the degenerate cyclotomic Hecke algebras 1 . The conclusion is exactly as claimed in Grojnowski's unpublished note $\mathrm{G}$; see $\S 4$ below for the precise statement. Unfortunately, as has been pointed out by Anton Cox, the argument given there is incomplete, so this fills a gap in the literature 2 . Actually, $\mathrm{G}$ was mainly concerned with cyclotomic Hecke algebras (not their rational degenerations). For these, it has also long been expected that the center consists of all symmetric polynomials in the Jucys-Murphy elements, but we still do not know how to prove this. Nevertheless, Lyle and Mathas [LM have recently managed to solve the problem of classifying the blocks of the cyclotomic Hecke algebras too, using the analogue of Jantzen's sum formula for cyclotomic $q$-Schur algebras from [JM].

Now we further specialize to the case that $F=\mathbb{C}$. Let $\mu=\left(\mu_{1}, \ldots, \mu_{l}\right)$ be an l-tuple of positive integers summing to $n$. Let $\mathfrak{g}=\mathfrak{g l}_{n}(\mathbb{C})$ and let $\mathfrak{p}$ be the standard parabolic subalgebra with block diagonal Levi subalgebra $\mathfrak{h}=\mathfrak{g l}_{\mu_{1}}(\mathbb{C}) \oplus \cdots \oplus \mathfrak{g l}_{\mu_{l}}(\mathbb{C})$. Let $\mathcal{O}^{\mu}$ be the category of all finitely generated $\mathfrak{g}$-modules which are locally finite as $\mathfrak{p}$-modules and integrable as $\mathfrak{h}$-modules, i.e. they lift to rational representations of $H=G L_{\mu_{1}}(\mathbb{C}) \times \cdots \times G L_{\mu_{l}}(\mathbb{C})$. This is the usual parabolic analogue of the BGG category $\mathcal{O}$, except that we are only allowing modules with integral weights/central characters. The category $\mathcal{O}^{\mu}$ decomposes as

$$
\mathcal{O}^{\mu}=\bigoplus_{\nu} \mathcal{O}_{\nu}^{\mu}
$$

where the direct sum is over integral central characters $\nu: Z(\mathfrak{g}) \rightarrow \mathbb{C}$ of the universal enveloping algebra $U(\mathfrak{g})$, and $\mathcal{O}_{\nu}^{\mu}$ is the full subcategory of $\mathcal{O}^{\mu}$ consisting of modules with generalized central character $\nu$. Our next result, also ultimately a consequence of Theorem 1, is an essential ingredient in both of the articles [B2] and

\footnotetext{
${ }^{1}$ In an earlier version of this article, we also explained that Theorem 1 implies the following assertion describing the blocks of degenerate affine Hecke algebras: two irreducible $H_{d}$-modules belong to the same block if and only if they have the same central character. However, Iain Gordon has pointed out that this already follows directly by a general result of Müller [BG] III.9.2], since $H_{d}$ is finite as a module over its center.

${ }^{2}$ The same mistake also appears in the proof of [BK1, Corollary 8.13] dealing with the twisted case. In the degenerate case in that setting, the appropriate analogue of Theorem 1 needed to fill the gap has recently been announced by Ruff $[\mathrm{R}]$ for all odd levels. In level one the result already appears in BK2 Theorem 3.2].
} 
[S], which give quite different (and independent) proofs of a conjecture of Khovanov $[\mathrm{Kh}$, Conjecture 3]. Recall that the center $Z(\mathcal{C})$ of an additive category $\mathcal{C}$ is the commutative ring consisting of all natural transformations from the identity functor to itself. For example, if $\mathcal{C}$ is the category of finite dimensional modules over a finite dimensional algebra $C$, then $Z(\mathcal{C})$ is canonically isomorphic to the center of the algebra $C$ itself.

Theorem 2. For any integral central character $\nu$, the natural map

$$
m_{\nu}^{\mu}: Z(\mathfrak{g}) \rightarrow Z\left(\mathcal{O}_{\nu}^{\mu}\right)
$$

sending $z \in Z(\mathfrak{g})$ to the natural transformation defined by left multiplication by $z$ is a surjective algebra homomorphism. Moreover, the dimension of $Z\left(\mathcal{O}_{\nu}^{\mu}\right)$ is the same as the number of isomorphism classes of irreducible modules in $\mathcal{O}_{\nu}^{\mu}$.

The category $\mathcal{O}_{\nu}^{\mu}$ is equivalent to the category of finite dimensional modules over a finite dimensional algebra, e.g. one can take the endomorphism algebra of a minimal projective generator. Hence, two irreducible modules in $\mathcal{O}_{\nu}^{\mu}$ belong to the same block if and only if they have the same central character with respect to $Z\left(\mathcal{O}_{\nu}^{\mu}\right)$. By definition, all irreducible modules in $\mathcal{O}_{\nu}^{\mu}$ have the same central character with respect to $Z(\mathfrak{g})$. So Theorem 2 implies that all the irreducible modules in $\mathcal{O}_{\nu}^{\mu}$ belong to the same block. This proves that the above decomposition of $\mathcal{O}^{\mu}$ (defined by central characters with respect to $Z(\mathfrak{g}))$ coincides with its decomposition into blocks (defined by linkage classes of irreducible modules). This coincidence is a special feature of type A. For an arbitrary semisimple Lie algebra and any parabolic subalgebra, [Ja, Corollar 4] (proved using the sum formula from [Ja, Satz 2]) implies for any regular integral central character $\nu: Z(\mathfrak{g}) \rightarrow \mathbb{C}$ that the full subcategory of parabolic category $\mathcal{O}$ consisting of modules with generalized central character $\nu$ is always a single block. However, for singular integral central characters in types other than type A, Platt $[\mathrm{P}$ has given counterexamples showing that the subcategory defined by the given central character can decompose as a sum of more than one block in general; for types $\mathrm{B}$ and $\mathrm{C}$ one can already find such examples in $\mathrm{ES}$.

The remainder of the article is organized as follows. There is a natural filtration on the algebra $H_{d}^{f}$ with respect to which the associated graded algebra $\operatorname{gr} H_{d}^{f}$ is the twisted tensor product of the level $l$ truncated polynomial algebra $R\left[x_{1}, \ldots, x_{d}\right] /\left(x_{1}^{l}, \ldots, x_{d}^{l}\right)$ by the group algebra $R S_{d}$ of the symmetric group. In section 2 , we compute the center of this associated graded algebra directly, giving the crucial upper bound on the size of $Z\left(H_{d}^{f}\right)$ since we obviously have that $\operatorname{gr} Z\left(H_{d}^{f}\right) \subseteq Z\left(\operatorname{gr} H_{d}^{f}\right)$. There are then several different ways to show that this upper bound is actually attained. The approach followed in section 3 is to simply write down enough linearly independent central elements in $H_{d}^{f}$. This has the advantage of yielding at the same time an explicit basis for $Z\left(H_{d}^{f}\right)$ which is a generalization of Murphy's basis for $Z\left(R S_{n}\right)$ constructed in the proof of [M, 1.9]. In section 4, we discuss the classification of the blocks of $H_{d}^{f}$ in more detail. In particular, we compute the dimension of the center of each block, refining Theorem 1, which gives the dimension of the center of the whole algebra. Finally, in section 5 we deduce the results about parabolic category $\mathcal{O}$ by exploiting the Schur-Weyl duality for higher levels from BK3, which reduces many questions about the category $\mathcal{O}^{\mu}$ to the degenerate cyclotomic Hecke algebras $H_{d}^{f}$ for $f(x)=\left(x-\mu_{1}\right) \cdots\left(x-\mu_{l}\right)$. 


\section{The Center of the ASsociated GRADED ALgebra}

We fix an integer $l \geq 1$ and a commutative ring $R$. Let $R_{l}\left[x_{1}, \ldots, x_{d}\right]$ denote the level $l$ truncated polynomial algebra, that is, the quotient of the polynomial algebra $R\left[x_{1}, \ldots, x_{d}\right]$ by the relations $x_{1}^{l}=\cdots=x_{d}^{l}=0$. The symmetric group $S_{d}$ acts on $R_{l}\left[x_{1}, \ldots, x_{d}\right]$ by algebra automorphisms so that $w \cdot x_{i}=x_{w i}$ for each $i$ and $w \in S_{d}$. We view the resulting twisted tensor product algebra $R_{l}\left[x_{1}, \ldots, x_{d}\right] \otimes R S_{d}$ as a graded algebra with each $x_{i}$ in degree 1 and all elements of $S_{d}$ in degree 0 . The goal in this section is to compute the center of this algebra explicitly. We remark that the algebra $R_{l}\left[x_{1}, \ldots, x_{d}\right] \otimes R S_{d}$ can be viewed as a degeneration of the group algebra $R\left(C_{l} 2 S_{d}\right)$ of the wreath product of the symmetric group and the cyclic group of order $l$. It is well known that the conjugacy classes of $C_{l} \prec S_{d}$ are parametrized by certain multipartitions; see [Mac, p. 170] or [W]. With this in mind the results in this section should not be too surprising.

Let $Q_{d}$ denote the centralizer of $R_{l}\left[x_{1}, \ldots, x_{d}\right]$ in $R_{l}\left[x_{1}, \ldots, x_{d}\right] \otimes R S_{d}$. The symmetric group $S_{d}$ acts on $Q_{d}$ by conjugation, i.e. $w \cdot z=w z w^{-1}$. It is obvious that the center of $R_{l}\left[x_{1}, \ldots, x_{d}\right] \otimes R S_{d}$ is just the set of fixed points

$$
Z\left(R_{l}\left[x_{1}, \ldots, x_{d}\right] \otimes R S_{d}\right)=Q_{d}^{S_{d}} .
$$

We are going first to describe an explicit basis for $Q_{d}$ from which it will be easy to determine the $S_{d}$-fixed points, hence the center.

For $r \geq 0$ and any set $I=\left\{i_{1}, \ldots, i_{a}\right\}$ of $a$ distinct numbers chosen from $\{1, \ldots, d\}$, let

$$
h_{r}(I)=h_{r}\left(i_{1}, \ldots, i_{a}\right):=\sum_{\substack{0 \leq r_{1}, \ldots, r_{a}<l \\ r_{1}+\cdots+r_{a}=(a-1)(l-1)+r}} x_{i_{1}}^{r_{1}} \cdots x_{i_{a}}^{r_{a}} \in R_{l}\left[x_{1}, \ldots, x_{d}\right],
$$

the $((a-1)(l-1)+r)$ th complete symmetric function in the variables $x_{i_{1}}, \ldots, x_{i_{a}}$. By the pigeonhole principle, $h_{r}(I)$ is zero if $r \geq l$, and, moreover, $h_{l-1}(I)=$ $x_{i_{1}}^{l-1} \cdots x_{i_{a}}^{l-1}$.

Lemma 2.1. Let $I, J$ be any two subsets of $\{1, \ldots, d\}$ with $c=|I \cap J|>0$. For any $r, s \geq 0$, we have that $h_{r}(I) h_{s}(J)=l^{c-1} h_{r+s+(c-1)(l-1)}(I \cup J)$.

Proof. Suppose first that $I=\left\{i_{1}, \ldots, i_{a}, k\right\}$ and $J=\left\{j_{1}, \ldots, j_{b}, k\right\}$ with $I \cap J=\{k\}$. Then we have that

$$
\begin{aligned}
h_{r}(I) h_{s}(J) & =\sum_{\substack{r_{1}, \ldots, r_{a} \\
s_{1}, \ldots, s_{b}}} x_{i_{1}}^{r_{1}} \cdots x_{i_{a}}^{r_{a}} x_{k}^{a(l-1)+r-r_{1}-\cdots-r_{a}} x_{j_{1}}^{s_{1}} \cdots x_{j_{b}}^{s_{b}} x_{k}^{b(l-1)+s-s_{1}-\cdots-s_{b}} \\
& =\sum_{\substack{r_{1}, \ldots, r_{a} \\
s_{1}, \ldots, s_{b}}} x_{i_{1}}^{r_{1}} \cdots x_{i_{a}}^{r_{a}} x_{j_{1}}^{s_{1}} \cdots x_{j_{b}}^{s_{b}} x_{k}^{(a+b)(l-1)+r+s-r_{1}-\cdots-r_{a}-s_{1}-\cdots-s_{b}} \\
& =h_{r+s}(I \cup J) .
\end{aligned}
$$

This proves the lemma in the case $c=1$. Next we take $i \neq j$ and note that

$$
\begin{aligned}
h_{0}(i, j) h_{0}(i, j) & =\sum_{r, s} x_{i}^{r} x_{j}^{l-1-r} x_{j}^{s} x_{i}^{l-1-s} \\
& =\sum_{r, s} x_{i}^{l-1+r-s} x_{j}^{l-1+s-r}=l x_{i}^{l-1} x_{j}^{l-1}=l h_{l-1}(i, j) .
\end{aligned}
$$


Finally, take $I=\left\{i_{1}, \ldots, i_{a}, k_{1}, \ldots, k_{c}\right\}, J=\left\{j_{1}, \ldots, j_{b}, k_{1}, \ldots, k_{c}\right\}$ for $c \geq 2$ and assume that $I \cap J=\left\{k_{1}, \ldots, k_{c}\right\}$. Using the preceeding two formulae, we get that

$$
\begin{aligned}
h_{r}(I) h_{s}(J)= & h_{r}\left(i_{1}, \ldots, i_{a}, k_{1}\right) h_{0}\left(k_{1}, k_{2}\right) \cdots h_{0}\left(k_{c-1}, k_{c}\right) \\
& \quad \times h_{0}\left(k_{1}, k_{2}\right) \cdots h_{0}\left(k_{c-1}, k_{c}\right) h_{s}\left(j_{1}, \ldots, j_{b}, k_{1}\right) \\
= & l^{c-1} h_{r+s+(c-1)(l-1)}\left(i_{1}, \ldots, i_{a}, j_{1}, \ldots, j_{b}, k_{1}, \ldots, k_{c}\right) \\
= & l^{c-1} h_{r+s+(c-1)(l-1)}(I \cup J) .
\end{aligned}
$$

This is what we wanted.

Now let $A=\left(i_{1} \cdots i_{a}\right)$ be an $a$-cycle in $S_{d}$. Write $h_{r}(A)$ for $h_{r}\left(i_{1}, \ldots, i_{a}\right)$. Given another cycle $B=\left(j_{1} \cdots j_{b}\right)$, write $A \cup B$ and $A \cap B$ for the sets $\left\{i_{1}, \ldots, i_{a}\right\} \cup$ $\left\{j_{1}, \ldots, j_{b}\right\}$ and $\left\{i_{1}, \ldots, i_{a}\right\} \cap\left\{j_{1}, \ldots, j_{b}\right\}$, respectively. Let

$$
A^{(r)}:=h_{r}(A) A \in R_{l}\left[x_{1}, \ldots, x_{d}\right] \otimes R S_{d},
$$

which we call a cycle of color $r$. As before, we have that $A^{(r)}=0$ for $r \geq l$, so we need only consider colors from the set $\{0,1, \ldots, l-1\}$. In the case of 1 -cycles, we have that $(i)^{(r)}=x_{i}^{r}$, so 1 -cycles of color 0 are trivial.

Lemma 2.2. Let $r, s \geq 0$ be colors and $A$ and $B$ be cycles in $S_{d}$. Let $c=|A \cap B|$.

(i) If $c=0$, then $A^{(r)} B^{(s)}=B^{(s)} A^{(r)}$, i.e. disjoint colored cycles commute.

(ii) If $c=1$ (in which case the product $A B$ is a single cycle), then

$$
A^{(r)} B^{(s)}=(A B)^{(r+s)} .
$$

(iii) If $c \geq 2$, then

$$
A^{(r)} B^{(s)}=\delta_{r+s, 0} l^{c-1} h_{(c-1)(l-1)}(A \cup B) A B .
$$

Proof. (i) Obvious.

(ii) Say $A=\left(i_{1} \cdots i_{a} k\right)$ and $B=\left(j_{1} \cdots j_{b} k\right)$. By Lemma 2.1, we have that

$$
\begin{aligned}
A^{(r)} B^{(s)} & =h_{r}\left(i_{1}, \ldots, i_{a}, k\right) A h_{s}\left(j_{1}, \ldots, j_{b}, k\right) B \\
& =h_{r}\left(i_{1}, \ldots, i_{a}, k\right) h_{s}\left(j_{1}, \ldots, j_{b}, i_{1}\right) A B \\
& =h_{r+s}\left(i_{1}, \ldots, i_{a}, j_{1}, \ldots, j_{b}, k\right) A B=(A B)^{(r+s)}
\end{aligned}
$$

as required.

(iii) Arguing exactly as in (ii), we get that

$$
A^{(r)} B^{(s)}=l^{c-1} h_{r+s+(c-1)(l-1)}(A \cup B) A B .
$$

Now observe that $h_{r+s+(c-1)(l-1)}(A \cup B)$ is zero unless $r+s=0$.

Now we are going to consider products of colored cycles. Using Lemma 2.2, it is easy to see that any such product is either zero or else it can be rewritten as some power of $l$ times a product of disjoint colored cycles, meaning a product $A_{1}^{\left(r_{1}\right)} \ldots A_{m}^{\left(r_{m}\right)}$ where $A_{1}, \ldots, A_{m}$ are disjoint cycles in $S_{d}$ and $0 \leq r_{1}, \ldots, r_{m}<l$ are some colors. Moreover, two such products of disjoint colored cycles are equal if and only if one can be obtained from the other by reordering the disjoint colored cycles and adding/removing some 1-cycles of color 0. For example,

$$
\begin{aligned}
(123)^{(4)}(792)^{(1)} & =((123)(792))^{(5)}=(12793)^{(5)}=(12793)^{(5)}(4)^{(0)}, \\
(123)^{(4)}(7921)^{(1)} & =0=(123)^{(0)}(79321)^{(0)}(\operatorname{assuming} l>1), \\
(123)^{(0)}(7921)^{(0)} & =l\left(x_{1} x_{2} x_{3} x_{7} x_{9}\right)^{l-1}(123)(7921)=l(1793)^{(l-1)}(2)^{(l-1)} .
\end{aligned}
$$


Theorem 2.3. The set of all products of disjoint colored cycles is a basis for $Q_{d}$. In particular, it is a free $R$-module of rank

$$
\sum \frac{d !}{r_{1} ! r_{2} ! \cdots}\left(\frac{l}{1}\right)^{r_{1}}\left(\frac{l}{2}\right)^{r_{2}} \cdots
$$

summing over all partitions $\left(1^{r_{1}} 2^{r_{2}} \cdots\right)$ of $d$.

Proof. Observe by applying Lemma 2.2 with $B^{(s)}=(i)^{(1)}=x_{i}$ that every colored cycle $A^{(r)}$ belongs to the algebra $Q_{d}$. Hence all products of colored cycles belong to $Q_{d}$. Moreover, any product of colored cycles is a linear combination of products of disjoint colored cycles, and the set of all products of disjoint colored cycles is linearly independent. It just remains to show that $Q_{d}$ is spanned by all products of colored cycles.

Suppose to start with that $A=\left(i_{1} \cdots i_{a}\right)$ is an $a$-cycle in $S_{d}$ and that

$$
z=\sum_{\substack{0 \leq r_{1}, \ldots, r_{a}<l \\ r_{1}+\cdots+r_{a}=k}} c_{r_{1}, \ldots, r_{a}} x_{i_{1}}^{r_{1}} \cdots x_{i_{a}}^{r_{a}} A
$$

is a non-zero homogeneous element of $Q_{d}$ of degree $k \geq 0$ for some coefficients $c_{r_{1}, \ldots, r_{a}} \in R$. We claim that $z$ is a scalar multiple of $A^{(r)}$ for some $0 \leq r<$ $l$ (in which case $k=(a-1)(l-1)+r)$. To see this, equating coefficients of $x_{i_{1}}^{r_{1}} \cdots x_{i_{j}}^{r_{j}+1} \cdots x_{i_{a}}^{r_{a}} A$ in the equation $x_{i_{j}} z=z x_{i_{j}}$ gives that

$$
c_{r_{1}, \ldots, r_{j}, r_{j+1}, \ldots, r_{a}}=c_{r_{1}, \ldots, r_{j}+1, r_{j+1}-1, \ldots, r_{a}}
$$

whenever $r_{j}<l-1$ for some $j=1, \ldots, a-1$, interpreting the right hand side as zero in case $r_{j+1}=0$. If $k=(a-1)(l-1)+r$ for $0 \leq r<l$, we deduce from this that all the coefficents $c_{r_{1}, \ldots, r_{a}}$ are equal to $c_{l-1, \ldots, l-1, r}$, hence $z$ is a scalar multiple of $A^{(r)}$. Otherwise, we can write $k=m(l-1)+r$ for some $m \leq$ $a-2$ and $0 \leq r<l-1$ and get that all the coefficients $c_{r_{1}, \ldots, r_{a}}$ are equal to $c_{l-1, \ldots, l-1, r, 0,0, \ldots, 0}=c_{l-1, \ldots, l-1, r+1,-1,0, \ldots, 0}=0$, contradicting the assumption that $z \neq 0$.

Now take an element $f w \in Q_{d}$ for $w \in S_{d}$ and a homogeneous polynomial $f \in R_{l}\left[x_{1}, \ldots, x_{d}\right]$. Write $w=A_{1} \cdots A_{m}$ as a product of disjoint cycles, none of which are 1-cycles. We show by induction on $m$ that $f w$ is a linear combination of products of colored cycles. The base case $m=0$ is clear, as then $w=1$. For the induction step, suppose that $m \geq 1$ and $A_{m}=\left(i_{1} \cdots i_{a}\right)$. Let $I=\left\{i_{1}, \ldots, i_{a}\right\}$ and $J=\{1, \ldots, d\} \backslash I$. We can write $f=\sum_{s=1}^{t} f_{s} g_{s}$ for homogeneous polynomials $f_{1}, \ldots, f_{t} \in R_{l}\left[x_{i} \mid i \in I\right]$ and linearly independent homogeneous polynomials $g_{1}, \ldots, g_{t} \in R_{l}\left[x_{j} \mid j \in J\right]$. Equating coefficients of $g_{s} A_{1} \cdots A_{m-1}$ in the equations $x_{i} f w=f w x_{i}$ for each $i \in I$, we deduce that each $f_{s} A_{m}$ belongs to $Q_{d}$. Hence by the previous paragraph each $f_{s} A_{m}$ is a scalar multiple of $A_{m}^{(r)}$ for some $0 \leq r<l-1$. This shows that $f w=\sum_{r=0}^{l-1} h_{r} A_{1} \cdots A_{m-1} A_{m}^{(r)}$ for homogeneous polynomials $h_{r} \in$ $R_{l}\left[x_{j} \mid j \in J\right]$. Equating coefficients of $A_{m}^{(r)}$ in the equations $x_{j} f w=f w x_{j}$ for each $j \in J$, we deduce that each $h_{r} A_{1} \cdots A_{m-1}$ belongs to $Q_{d}$. Hence by the induction hypothesis each $h_{r} A_{1} \cdots A_{m-1}$ is a linear combination of products of colored cycles. Hence $f w$ is too.

Finally, take an arbitrary homogeneous element $\sum_{w \in S_{d}} f_{w} w \in Q_{d}$, for polynomials $f_{w} \in R_{l}\left[x_{1}, \ldots, x_{d}\right]$. We have for each $i$ that $\sum_{w \in S_{d}} x_{i} f_{w} w=\sum_{w \in S_{d}} x_{w i} f_{w} w$. Equating coefficients gives that $x_{i} f_{w} w=x_{w i} f_{w} w=f_{w} w x_{i}$ for each $i$ and $w$. Hence 
each $f_{w} w$ belongs to $Q_{d}$. So by the previous paragraph each $f_{w} w$ is a linear combination of products of colored cycles. This completes the proof.

For a partition $\lambda=\left(\lambda_{1} \geq \lambda_{2} \geq \cdots\right)$ we write $|\lambda|$ for $\lambda_{1}+\lambda_{2}+\cdots$ and $\ell(\lambda)$ for its length, that is, the number of non-zero parts. By an l-multipartition of $d$ we mean a tuple $\lambda=\left(\lambda^{(1)}, \lambda^{(2)}, \ldots, \lambda^{(l)}\right)$ of partitions such that $\left|\lambda^{(1)}\right|+\cdots+$ $\left|\lambda^{(l)}\right|=d$. Let $\mathcal{M}_{d}(l)$ denote the set of all $l$-multipartitions of $d$. Given a product $z=A_{1}^{\left(r_{1}\right)} \cdots A_{m}^{\left(r_{m}\right)}$ of disjoint colored cycles in $Q_{d}$, where each $A_{i}$ is an $a_{i}$-cycle, we can add extra 1-cycles of color 0 if necessary to assume that $a_{1}+\cdots+a_{m}=d$. Define the cycle type of $z$ to be the $l$-multipartition $\lambda=\left(\lambda^{(1)}, \ldots, \lambda^{(l)}\right)$ of $d$ defined by declaring that $\lambda^{(r)}$ is the partition whose parts consist of all the $a_{i}$ such that $r_{i}=r-1$. For $\lambda=\left(\lambda^{(1)}, \ldots, \lambda^{(l)}\right) \in \mathcal{M}_{d}(l)$, let $z_{d}(\lambda)$ denote the sum of all products of disjoint colored cycles in $Q_{d}$ of cycle type $\lambda$.

Theorem 2.4. The elements $\left\{z_{d}(\lambda) \mid \lambda \in \mathcal{M}_{d}(l)\right\}$ form a basis for the center of $R_{l}\left[x_{1}, \ldots, x_{d}\right] \otimes R S_{d}$. In particular, $Z\left(R_{l}\left[x_{1}, \ldots, x_{d}\right] \otimes R S_{d}\right)$ is a free $R$-module of $\operatorname{rank}\left|\mathcal{M}_{d}(l)\right|$.

Proof. As we remarked at the beginning of the section, the center of $R_{l}\left[x_{1}, \ldots, x_{d}\right] \otimes$ $R S_{d}$ is the set of fixed points of $S_{d}$ on $Q_{d}$. Given a colored cycle $A^{(r)}=\left(i_{1} \cdots i_{a}\right)$ and $w \in S_{d}$, we have that

$$
w \cdot A^{(r)}=(w \cdot A)^{(r)}=\left(w i_{1} \cdots w i_{a}\right)^{(r)} .
$$

So the action of $S_{d}$ on $Q_{d}$ is the linear action induced by a permutation action on the basis from Theorem 2.3. It just remains to observe that two products of disjoint colored cycles lie in the same $S_{d}$-orbit if and only if they have the same cycle type, and the $z_{d}(\lambda)$ 's are simply the orbit sums.

Corollary 2.5. If $d$ ! is invertible in $R$, then the center of $R_{l}\left[x_{1}, \ldots, x_{d}\right] \otimes R S_{d}$ is generated by the elements

$$
z_{d}\left(a^{(r)}\right):=\sum_{\text {all a-cycles }} A_{A \in S_{d}}^{(r)}
$$

for all $0 \leq r<l$ and $1 \leq a \leq d$.

Proof. Take a multipartition $\lambda=\left(\lambda^{(1)}, \ldots, \lambda^{(l)}\right) \in \mathcal{M}_{d}(l)$. Consider the product of the elements $z_{d}\left(a^{(r-1)}\right)$ over all $r=1, \ldots, l$ and all non-zero parts $a$ of $\lambda^{(r)}$. It gives an invertible scalar multiple of $z_{d}(\lambda)$ modulo lower terms.

In the remainder of the section, we are going to construct another basis for $Z\left(R_{l}\left[x_{1}, \ldots, x_{d}\right] \otimes R S_{d}\right)$ which is a generalization of the basis for the center of $R S_{n}$ constructed by Murphy in the proof of [M, 1.9]. Given $k \geq 0$ and $1 \leq i \leq d$, write $k=(a-1) l+r$ for $a \geq 1$ and $0 \leq r<l$, then set

$$
y_{i}(k):=\sum_{\substack{1 \leq i_{1}, \ldots, i_{a-1}<i \\ i_{1}, \ldots, i_{a-1} \text { distinct }}}\left(i_{1} \cdots i_{a-1} i\right)^{(r)},
$$

an element of degree $(a-1)(l-1)+r$. For example, $y_{i}(k)=0$ if $k \geq i l$ and $y_{i}(r)=(i)^{(r)}=x_{i}^{r}$ for $0 \leq r<l$. Of particular importance, we have that

$$
y_{i}(l)=\sum_{j=1}^{i-1}(j i)^{(0)},
$$

which we call the $i$ th colored Jucys-Murphy element. 
Lemma 2.6. For any $1 \leq i \leq d$ and $p \geq 0$, we have that

$$
y_{i}(l)^{p}=y_{i}(p l)+(*)
$$

where $(*)$ is a linear combination of products $A_{1}^{(l-1)} \cdots A_{m}^{(l-1)}$ for disjoint cycles $A_{1}, \ldots, A_{m}$ in $S_{i}$ such that $A_{1}$ involves $i$ and $\left|A_{1} \cup \cdots \cup A_{m}\right| \leq p$.

Proof. Induction exercise using Lemma 2.2 .

For a partition $\lambda=\left(\lambda_{1} \geq \lambda_{2} \geq \cdots\right)$, let $\lambda / l:=\left(\left\lfloor\lambda_{1} / l\right\rfloor \geq\left\lfloor\lambda_{2} / l\right\rfloor \geq \cdots\right)$. We are going to use partitions belonging to the set

$$
\mathcal{P}_{d}(l)=\{\lambda|\ell(\lambda)+| \lambda / l \mid \leq d\}
$$

to parametrize our new basis. To start with note that $\left|\mathcal{P}_{d}(l)\right|=\left|\mathcal{M}_{d}(l)\right|$, so this set is of the correct size. Indeed, there is a bijection

$$
\varphi: \mathcal{M}_{d}(l) \rightarrow \mathcal{P}_{d}(l),
$$

defined as follows. Suppose that $\lambda=\left(\lambda^{(1)}, \ldots, \lambda^{(l)}\right) \in \mathcal{M}_{d}(l)$ where $\lambda^{(r)}=\left(\lambda_{1}^{(r)} \geq\right.$ $\left.\cdots \geq \lambda_{m_{r}}^{(r)}>0\right)$. Then $\varphi(\lambda)$ denotes the ordinary partition with parts $\left(\lambda_{i}^{(r)}-1\right) l+$ $r-1$ for all $1 \leq r \leq l$ and $1 \leq i \leq m_{r}$. It is easy to see that $\varphi(\lambda)$ belongs to $\mathcal{P}_{d}(l)$. Conversely, given $\mu=\left(\mu_{1} \geq \mu_{2} \geq \cdots\right) \in \mathcal{P}_{d}(l)$, there is a unique multipartition $\lambda=\left(\lambda^{(1)}, \ldots, \lambda^{(l)}\right)$ of $d$ such that $\varphi(\lambda)=\mu$ : the parts of $\lambda^{(r)}$ are the numbers $\left\lfloor\mu_{i} / l\right\rfloor+1$ for all $i=1, \ldots, d-|\mu / l|$ such that $\mu_{i} \equiv r-1(\bmod l)$. Hence $\varphi$ is indeed a bijection.

Since every element $\mu$ of $\mathcal{P}_{d}(l)$ is of length at most $d$, it can be thought of simply as a $d$-tuple of integers. Given two $d$-tuples $\mu=\left(\mu_{1}, \ldots, \mu_{d}\right)$ and $\nu=\left(\nu_{1}, \ldots, \nu_{d}\right)$ we write $\mu \sim \nu$ if one is obtained from the other by permuting the entries. For $\mu=\left(\mu_{1}, \ldots, \mu_{d}\right) \in \mathcal{P}_{d}(l)$, define

$$
m_{d}(\mu):=\sum_{\nu \sim \mu} y_{1}\left(\nu_{1}\right) \cdots y_{d}\left(\nu_{d}\right)
$$

This is a homogeneous element of $Q_{d}$ of degree $|\mu|-|\mu / l|$.

Theorem 2.7. The elements $\left\{m_{d}(\mu) \mid \mu \in \mathcal{P}_{d}(l)\right\}$ form a basis for the center of $R_{l}\left[x_{1}, \ldots, x_{d}\right] \otimes R S_{d}$.

Proof. Let us first check that $m_{d}(\mu)$ belongs to $Z\left(R_{l}\left[x_{1}, \ldots, x_{d}\right] \otimes R S_{d}\right)$. We just need to check that it commutes with each basic transposition $(i i+1)$. Obviously, $(i i+1)$ commutes with $y_{j}(m)$ if $j \neq i, i+1$. Therefore, it suffices to show for each $i=$ $1, \ldots, d-1$ and $k, m \geq 0$ that $(i i+1)$ commutes with both the elements $y_{i}(k) y_{i+1}(k)$ and $y_{i}(k) y_{i+1}(m)+y_{i}(m) y_{i+1}(k)$. For the first case, write $k=(a-1) l+r$ as usual. We have that

$$
y_{i}(k) y_{i+1}(k)=\sum_{\substack{1 \leq i_{1}, \ldots, i_{a-1}<i \\ 1 \leq j_{1}, \ldots, j_{a-1}<i+1}}\left(i_{1} \cdots i_{a-1} i\right)^{(r)}\left(j_{1} \cdots j_{a-1} i+1\right)^{(r)}
$$

where the sum is over distinct $i_{1}, \ldots, i_{a-1}$ and distinct $j_{1}, \ldots, j_{a-1}$. We split this sum into two pieces:

$$
\sum_{\substack{1 \leq i_{1}, \ldots, i_{a-1}<i \\ 1 \leq j_{1}, \ldots, j_{a-1}<i}}\left(i_{1} \cdots i_{a-1} i\right)^{(r)}\left(j_{1} \cdots j_{a-1} i+1\right)^{(r)}
$$


which clearly commutes with $(i i+1)$, and

$$
\sum_{b=1}^{a-1} \sum_{\substack{1 \leq i_{1}, \ldots, i_{a-1}<i \\ 1 \leq j_{1}, \ldots, j_{a-1}<i+1 \\ j_{b}=i}}\left(i_{1} \cdots i_{a-1} i\right)^{(r)}\left(j_{1} \cdots j_{b-1} i j_{b+1} \cdots j_{a-1} i+1\right)^{(r)}
$$

which also commutes with $(i i+1)$ by an application of Lemma 2.2. The second case is similar.

Now we compare the $m_{d}(\mu)$ 's with the basis from Theorem 2.4 For any $\lambda \epsilon$ $\mathcal{M}_{d}(l)$ define $\# \lambda$ to be $(d-z)$ where $z$ is the number of parts of $\lambda^{(1)}$ that equal 1 . We claim for $\lambda \in \mathcal{M}_{d}(l)$ with $\varphi(\lambda)=\mu$ that $m_{d}(\mu)=z_{d}(\lambda)+(*)$ where $(*)$ is a linear combination of $z_{d}(\nu)$ 's for $\nu \in \mathcal{M}_{d}(l)$ with $\# \nu<\# \lambda$. The theorem clearly follows from this claim and Theorem 2.4. To prove the claim, let $\mu=\left(\mu_{1} \geq \cdots \geq \mu_{h}>0\right)$ and write each $\mu_{i}$ as $\left(a_{i}-1\right) l+r_{i}$ as usual, so $\# \lambda=a_{1}+\cdots+a_{h}$. By definition, $m_{d}(\mu)$ is a sum of products of colored cycles of the form $z=A_{1}^{\left(r_{1}\right)} \cdots A_{h}^{\left(r_{h}\right)}$ where each $A_{i}$ is an $a_{i}$-cycle. If $A_{1}, \ldots, A_{h}$ happen to be disjoint cycles, then $z$ is of cycle type $\lambda$. Otherwise, $\left|A_{1} \cup \cdots \cup A_{h}\right|<\# \lambda$ so using Lemma 2.2 we can rewrite $z$ as a linear combination of products of disjoint colored cycles of cycle type $\nu \in \mathcal{M}_{d}(l)$ with $\# \nu<\# \lambda$. Combined with the first paragraph and Theorem 2.4 this shows that $m_{d}(\mu)=c z_{d}(\lambda)+(*)$ for some $c$. Finally, to show that $c=1$, consider the coefficient of one particular product of disjoint colored cycles of cycle type $\lambda$ in the expansion of $m_{d}(\mu)$.

\section{THE CENTER OF $H_{d}^{f}$}

We are ready to tackle the problem of computing the center of the degenerate cyclotomic Hecke algebra $H_{d}^{f}$, where $f(x)=x^{l}+c_{1} x^{l-1}+\cdots+c_{l} \in R[x]$ is a monic polynomial of degree $l$. Define a filtration

$$
\mathrm{F}_{0} H_{d}^{f} \subseteq \mathrm{F}_{1} H_{d}^{f} \subseteq \mathrm{F}_{2} H_{d}^{f} \subseteq \cdots
$$

of the algebra $H_{d}^{f}$ by declaring that $\mathrm{F}_{r} H_{d}^{f}$ is spanned by all $x_{i_{1}} \cdots x_{i_{s}} w$ for $0 \leq$ $s \leq r, 1 \leq i_{1}, \ldots, i_{s} \leq d$ and $w \in S_{d}$. So each $x_{i}$ is in filtered degree 1 and each $w \in S_{d}$ is in filtered degree 0 . Given an element $z \in \mathrm{F}_{r} H_{d}^{f}$, we write $\operatorname{gr}_{r} z$ for its canonical image in the $r$ th graded component $\operatorname{gr}_{r} H_{d}^{f}=\mathrm{F}_{r} H_{d}^{f} / \mathrm{F}_{r-1} H_{d}^{f}$ of the associated graded algebra gr $H_{d}^{f}=\bigoplus_{r \geq 0} \mathrm{gr}_{r} H_{d}^{f}$. By the PBW theorem for degenerate cyclotomic Hecke algebras [BK3, Lemma 3.5], this associated graded algebra gr $H_{d}^{f}$ can be identified with the twisted tensor product $R_{l}\left[x_{1}, \ldots, x_{d}\right] \otimes R S_{d}$ so that $\operatorname{gr}_{1} x_{i}$ is identified with $x_{i} \in R_{l}\left[x_{1}, \ldots, x_{d}\right] \otimes R S_{d}$ and $\operatorname{gr}_{0} w$ is identified with $w \in R_{l}\left[x_{1}, \ldots, x_{d}\right] \otimes R S_{d}$. To avoid confusion, we reserve the notations $x_{i}^{r}$ and $s_{i}$ from now on for the elements of $H_{d}^{f}$, always using the alternate notations $(i)^{(r)}$ and $(i i+1)$ for the corresponding elements of the associated graded algebra $R_{l}\left[x_{1}, \ldots, x_{d}\right] \otimes R S_{d}$.

Given an $R$-submodule $V$ of $H_{d}^{f}$, we can consider the induced filtration on $V$ defined by setting $\mathrm{F}_{r} V:=V \cap \mathrm{F}_{r} H_{d}^{f}$. The associated graded module $\operatorname{gr} V$ is canonically identified with an $R$-submodule of $R_{l}\left[x_{1}, \ldots, x_{d}\right] \otimes R S_{d}$, and for two submodules we have that $V=V^{\prime}$ if and only if $\operatorname{gr} V=\operatorname{gr} V^{\prime}$. Note also that

$$
\operatorname{gr} Z\left(H_{d}^{f}\right) \subseteq Z\left(R_{l}\left[x_{1}, \ldots, x_{d}\right] \otimes R S_{d}\right) .
$$


Hence if we can find elements $z_{1} \in \mathrm{F}_{i_{1}} Z\left(H_{d}^{f}\right), \ldots, z_{m} \in \mathrm{F}_{i_{m}} Z\left(H_{d}^{f}\right)$ with the property that $\operatorname{gr}_{i_{1}} z_{1}, \ldots, \mathrm{gr}_{i_{m}} z_{m}$ is a basis for $Z\left(R_{l}\left[x_{1}, \ldots, x_{d}\right] \otimes R S_{d}\right)$, then it follows immediately that $z_{1}, \ldots, z_{m}$ also is a basis for $Z\left(H_{d}^{f}\right)$. This is exactly what we are going to do. Recall the elements $y_{i}(k)$ and $m_{d}(\mu)$ of $R_{l}\left[x_{1}, \ldots, x_{d}\right] \otimes R S_{d}$ from the previous section.

Lemma 3.1. Assume that $1 \leq i \leq d$ and $k=(a-1) l+r$ for some $a \geq 1$ and $0 \leq r<l$. Then we have that $x_{i}^{k} \in \mathrm{F}_{(a-1)(l-1)+r} H_{d}^{f}$ and

$$
\operatorname{gr}_{(a-1)(l-1)+r} x_{i}^{k}= \begin{cases}y_{i}(k)+(*) & \text { if } r=0, \\ y_{i}(k) & \text { if } r>0,\end{cases}
$$

where $(*)$ denotes a linear combination of products of disjoint colored cycles of the form $A_{1}^{(l-1)} \cdots A_{m}^{(l-1)}$ such that $i \in A_{1} \cup \cdots \cup A_{m} \subseteq\{1, \ldots, i\}$ and $\left|A_{1} \cup \cdots \cup A_{m}\right| \leq$ $a-1$.

Proof. Assume to start with that $k=l$, i.e. $a=2, r=0$. We prove the lemma in this case by induction on $i=1, \ldots, d$. For the base case, we have that $x_{1}^{l}=$ $-c_{1} x_{1}^{l-1}-\cdots-c_{l}$, so it is in filtered degree $(l-1)$ and $\operatorname{gr}_{l-1} x_{1}^{l}=-c_{1}(1)^{(l-1)}=$ $y_{1}(l)-c_{1}(1)^{(l-1)}$. For the induction step, we have by the relations that

$$
x_{i+1}^{l}=s_{i} x_{i}^{l} s_{i}+\sum_{t=0}^{l-1} x_{i}^{t} x_{i+1}^{l-1-t} s_{i} .
$$

Hence by induction we get that $x_{i+1}^{l}$ is in filtered degree $(l-1)$ and

$$
\begin{aligned}
\operatorname{gr}_{l-1} x_{i+1}^{l} & =(i i+1)\left(y_{i}(l)-c_{1}(i)^{(l-1)}\right)(i i+1)+(i i+1)^{(0)} \\
& =y_{i+1}(l)-c_{1}(i+1)^{(l-1)}
\end{aligned}
$$

as we wanted.

Now assume that $k=(a-1) l$ for any $a \geq 1$, i.e. the case when $r=0$. By the previous paragraph, we have that $x_{i}^{k}=\left(x_{i}^{l}\right)^{a-1}$ is in filtered degree $(a-1)(l-1)$ and

$$
\operatorname{gr}_{(a-1)(l-1)} x_{i}^{k}=\left(y_{i}(l)-c_{1}(i)^{(l-1)}\right)^{a-1} .
$$

By Lemma 2.6 this equals $y_{i}(k)+(*)$ where $(*)$ is a linear combination of products of disjoint colored cycles of the form $A_{1}^{(l-1)} \cdots A_{m}^{(l-1)}$ such that $i \in A_{1} \cup \cdots \cup A_{m} \subseteq$ $\{1, \ldots, i\}$ and $\left|A_{1} \cup \cdots \cup A_{m}\right| \leq a-1$.

Finally, assume that $k=(a-1) l+r$ for $0<r<l$. Writing $x_{i}^{k}=\left(x_{i}^{(a-1) l}\right)\left(x_{i}^{r}\right)$ and using the previous paragraph and Lemma 2.2 gives the desired conclusion in this case.

For any $d$-tuple $\mu=\left(\mu_{1}, \ldots, \mu_{d}\right)$ of non-negative integers, let

$$
p_{d}(\mu):=\sum_{\nu \sim \mu} x_{1}^{\nu_{1}} \cdots x_{d}^{\nu_{d}} \in H_{d}^{f} .
$$

Since this is a symmetric polynomial in $x_{1}, \ldots, x_{d}$, it is automatically central. Theorem 1 from the introduction is a consequence of the following more precise result.

Theorem 3.2. For $\mu \in \mathcal{P}_{d}(l)$, we have that $p_{d}(\mu) \in \mathrm{F}_{r} Z\left(H_{d}^{f}\right)$ where $r=|\mu|-$ $|\mu / l|$. Moreover, $\operatorname{gr}_{r} p_{d}(\mu)=m_{d}(\mu)+(*)$ where $(*)$ is a linear combination of 
$m_{d}(\nu)$ 's for $\nu \in \mathcal{P}_{d}(l)$ with $|\nu / l|+\ell(\nu)<|\mu / l|+\ell(\mu)$. Hence, gr $Z\left(H_{d}^{f}\right)=$ $Z\left(R_{l}\left[x_{1}, \ldots, x_{d}\right] \otimes R S_{d}\right)$ and the elements

$$
\left\{p_{d}(\mu) \mid \mu \in \mathcal{P}_{d}(l)\right\}
$$

form a basis for $Z\left(H_{d}^{f}\right)$. In particular, $Z\left(H_{d}^{f}\right)$ is a free $R$-module of rank equal to the number of l-multipartitions of $d$.

Proof. Recall the bijection $\varphi: \mathcal{M}_{d}(l) \rightarrow \mathcal{P}_{d}(l)$ and also the notation $\# \lambda$ from the last paragraph of the proof of Theorem 2.7. We showed there for $\lambda \in \mathcal{M}_{d}(l)$ with $\varphi(\lambda)=\mu$ that $m_{d}(\mu)=z_{d}(\lambda)+(*)$ where $(*)$ is a linear combination of $z_{d}(\nu)$ 's with $\# \nu<\# \lambda$. Note $\# \lambda=|\mu / l|+\ell(\mu)$. So we get from this also that $z_{d}(\lambda)=m_{d}(\mu)+(*)$ where $(*)$ is a linear combination of $m_{d}(\nu)$ 's for $\nu \in \mathcal{P}_{d}(l)$ with $|\nu / l|+\ell(\nu)<|\mu / l|+\ell(\mu)$.

Now, by Lemma 3.1 and the definitions, $p_{d}(\mu)$ is in filtered degree $r=|\mu|-|\mu / l|$ and, moreover, $\operatorname{gr}_{r} p_{d}(\mu)=m_{d}(\mu)+(*)$ where $(*)$ is a linear combination of products of disjoint colored cycles $A_{1}^{\left(r_{1}\right)} \cdots A_{m}^{\left(r_{m}\right)}$ such that $\left|A_{1} \cup \cdots \cup A_{m}\right|<|\mu / l|+\ell(\mu)$. Since $\operatorname{gr}_{r} p_{d}(\mu)$ is central, it follows by Lemma 2.2 and Theorem 2.4 that $(*)$ can be rewritten as a linear combination of $z_{d}(\nu)$ 's with $\# \nu<\# \lambda$. Hence by the first paragraph it is also a linear combination of $m_{d}(\nu)$ 's with $|\nu / l|+\ell(\nu)<|\mu / l|+\ell(\mu)$. This proves that the elements

$$
\left\{\operatorname{gr}_{|\mu|-|\mu / l|} p_{d}(\mu) \mid \mu \in \mathcal{P}_{d}(l)\right\}
$$

form a basis for $Z\left(R_{l}\left[x_{1}, \ldots, x_{d}\right] \otimes R S_{d}\right)$. Now the theorem follows by the general principles discussed just before Lemma 3.1 .

Corollary 3.3. If $d$ ! is invertible in $R$, then the center of $H_{d}^{f}$ is generated by the power sums $x_{1}^{r}+\cdots+x_{d}^{r}$ for $1 \leq r \leq d$.

Proof. Under the assumption on $R$, it is well known that every symmetric polynomial in variables $x_{1}, \ldots, x_{d}$ can be expressed as a polynomial in the first $d$ power sums.

\section{THE BLOCKS OF $H_{d}^{f}$}

In this section, we replace the ground ring $R$ with a ground field $F$ over which we can factor $f(x)=\left(x-\mu_{1}\right) \cdots\left(x-\mu_{l}\right)$ for $\mu_{1}, \ldots, \mu_{l} \in F$. We will denote the algebra $H_{d}^{f}$ instead by $H_{d}^{\mu}$ where $\mu=\left(\mu_{1}, \ldots, \mu_{l}\right) \in F^{l}$. We point out that $F$ is a splitting field for the algebra $H_{d}^{\mu}$; one way to see this is to observe that the construction of the irreducible $H_{d}^{\mu}$-modules over the algebraic closure of $F$ from $\underline{\mathrm{K}}$, $\S 5.4]$ already makes sense over $F$ itself. Theorem 1 just proved shows in particular that the dimension of $Z\left(H_{d}^{\mu}\right)$ is equal to the number of $l$-multipartitions of $d$. The goal in this section is to refine this statement by computing the dimensions of the centers of the individual blocks.

Before we can even formulate the result, we need an explicit combinatorial parametrization of the blocks, or equivalently, the central characters of $H_{d}^{\mu}$. This is a well-known consequence of Theorem 1 . To start with we recall the classification of central characters of $H_{d}$ itself following [K, §4.2]. Given a tuple $\boldsymbol{i}=\left(i_{1}, \ldots, i_{d}\right) \in F^{d}$, write

$$
\chi(i): Z\left(H_{d}\right) \rightarrow F
$$


for the central character mapping a symmetric polynomial $f\left(x_{1}, \ldots, x_{d}\right)$ to $f\left(i_{1}, \ldots, i_{d}\right)$. Clearly, $\chi(\boldsymbol{i})=\chi(\boldsymbol{j})$ if and only if $\boldsymbol{i} \sim \boldsymbol{j}$, so this gives a parametrization of central characters of $H_{d}$ by the set $X_{d}$ of $\sim$-equivalence classes in $F^{d}$. Now we pass to the quotient $H_{d}^{\mu}$ of $H_{d}$. Since $Z\left(H_{d}\right)$ maps surjectively onto $Z\left(H_{d}^{\mu}\right)$ by Theorem 1 , the set of all central characters of $H_{d}^{\mu}$ is naturally parametrized by the subset

$$
X_{d}^{\mu}=\left\{\boldsymbol{i} \in X_{d} \mid \chi(\boldsymbol{i}): Z\left(H_{d}\right) \rightarrow F \text { factors through the quotient } Z\left(H_{d}^{\mu}\right)\right\}
$$

of $X_{d}$. Given $i \in X_{d}^{\mu}$ we will write $\bar{\chi}(\boldsymbol{i}): Z\left(H_{d}^{\mu}\right) \rightarrow F$ for the central character of $H_{d}^{\mu}$ induced by $\chi(\boldsymbol{i}): Z\left(H_{d}\right) \rightarrow F$. To complete the classification of blocks of $H_{d}^{\mu}$, it just remains to describe the subset $X_{d}^{\mu}$ combinatorially.

To do this, we must first construct enough central characters, which we do by considering Specht modules following [BK3, §6]. For a partition $\lambda$ of $d$, let $S_{\lambda}$ denote the corresponding Specht module for the symmetric group over the field $F$; we are deliberately being historically inaccurate here in referring to $S_{\lambda}$ as a Specht module as we actually mean the dual of the Specht module $S^{\lambda}$ introduced originally by James [J]. Given any $m \in F$, we can extend $S_{\lambda}$ to a module over the degenerate affine Hecke algebra $H_{d}$ so that $x_{1}$ acts by scalar multiplication by $m$. We denote the resulting $H_{d}$-module by $S_{\lambda}^{m}$. If $d=d^{\prime}+d^{\prime \prime}$, there is a natural embedding of $H_{d^{\prime}, d^{\prime \prime}}:=H_{d^{\prime}} \otimes H_{d^{\prime \prime}}$ into $H_{d}$, so it makes sense to define the product

$$
M^{\prime} \circ M^{\prime \prime}=H_{d} \otimes_{H_{d^{\prime}, d^{\prime \prime}}}\left(M^{\prime} \otimes M^{\prime \prime}\right)
$$

of an $H_{d^{\prime}}$-module $M^{\prime}$ and an $H_{d^{\prime \prime}}$-module $M^{\prime \prime}$, where $\otimes$ denotes the outer tensor product. Given an l-multipartition $\lambda=\left(\lambda^{(1)}, \lambda^{(2)}, \ldots, \lambda^{(l)}\right)$ of $d$, the $H_{d}$-module

$$
S_{\lambda}^{\mu}:=S_{\lambda(1)}^{\mu_{1}} \circ \cdots \circ S_{\lambda^{(l)}}^{\mu_{l}}
$$

factors through the quotient $H_{d}^{\mu}$ to give a well-defined $H_{d}^{\mu}$-module. This is the Specht module parametrized by the multipartition $\lambda$.

Let us compute the central character of the Specht module $S_{\lambda}^{\mu}$. Note that if $M^{\prime}$ is an $H_{d^{\prime}}$-module of central character $\chi\left(\boldsymbol{i}^{\prime}\right)$ and $M^{\prime \prime}$ is an $H_{d^{\prime \prime}}$-module of central character $\chi\left(\boldsymbol{i}^{\prime \prime}\right)$, then $M^{\prime} \circ M^{\prime \prime}$ is of central character $\chi\left(\boldsymbol{i}^{\prime} \circ \boldsymbol{i}^{\prime \prime}\right)$ where $\boldsymbol{i}^{\prime} \circ \boldsymbol{i}^{\prime \prime}$ denotes the concatenation $\left(i_{1}^{\prime}, \ldots, i_{d^{\prime}}^{\prime}, i_{1}^{\prime \prime}, \ldots, i_{d^{\prime \prime}}^{\prime}\right)$. This reduces to the problem of computing the central character simply of $S_{\lambda}^{m}$ for a partition $\lambda$ of $d$ and a scalar $m \in F$, which is well known: for each $i, j \geq 1$ fill the box in the $i$ th row and $j$ th column of the Young diagram of $\lambda$ with the residue $(m+j-i)$; then $S_{\lambda}^{\mu}$ is of central character parametrized by the tuple $\boldsymbol{i}_{\lambda}^{\mu}$ obtained by reading off the residues in all the boxes in some order. For example, if $\lambda=(4,2,1)$ and $m=5$, then the residues are

\begin{tabular}{|l|l|l|l|}
\hline 5 & 6 & 7 & 8 \\
\cline { 1 - 2 } 4 & 5 & \multicolumn{3}{|c}{} \\
\cline { 1 - 2 } 3 & \multicolumn{3}{|c}{} \\
\cline { 1 - 1 } & &
\end{tabular}

and $\boldsymbol{i}_{\lambda}^{m} \sim(5,6,7,8,4,5,3)$. Given $\lambda=\left(\lambda^{(1)}, \ldots, \lambda^{(l)}\right)$, we deduce that $S_{\lambda}^{\mu}$ is of central character parametrized by

$$
i_{\lambda}^{\mu}=i_{\lambda^{(1)}}^{\mu_{1}} \circ \cdots \circ i_{\lambda^{(l)}}^{\mu_{l}} .
$$

In this way, we have proved the existence of many central characters of $H_{d}^{\mu}$, namely, the central characters $\left\{\bar{\chi}\left(i_{\lambda}^{\mu}\right) \mid \lambda \in \mathcal{M}_{d}(l)\right\}$.

To show that this construction gives all the central characters, we proceed as in finite group theory. Let $\bar{R}$ be a Noetherian domain with maximal ideal $\overline{\mathfrak{m}}$ such that 
$F=\bar{R} / \overline{\mathfrak{m}}$ and the field of fractions of $\bar{R}$ is of characteristic 0 . Let $\hat{\mu}_{1}, \ldots, \hat{\mu}_{l} \in \bar{R}$ be lifts of the parameters $\mu_{1}, \ldots, \mu_{l} \in F$. Let $R$ be the completion of the polynomial algebra $\bar{R}\left[\tau_{1}, \ldots, \tau_{l}\right]$ with respect to the maximal ideal generated by $\overline{\mathfrak{m}}$ and $\tau_{1}-$ $\hat{\mu}_{1}, \ldots, \tau_{l}-\hat{\mu}_{l}$. We still have that $F=R / \mathfrak{m}$, where $\mathfrak{m}$ is the unique maximal ideal of $R$. Also, let $K$ be the field of fractions of $R$. Letting $\tau=\left(\tau_{1}, \ldots, \tau_{l}\right)$, define $H_{d}^{\tau}$ and $\mathcal{H}_{d}^{\tau}$ to be the degenerate cyclotomic Hecke algebras defined by the polynomial $f(x)=\left(x-\tau_{1}\right) \cdots\left(x-\tau_{l}\right)$ over the field $K$ and over the ring $R$, respectively. In view of the PBW theorem for degenerate cyclotomic Hecke algebras, $H_{d}^{\tau}$ is naturally isomorphic to $K \otimes_{R} \mathcal{H}_{d}^{\tau}$, and $H_{d}^{\mu}$ is naturally isomorphic to $F \otimes_{R} \mathcal{H}_{d}^{\tau}$, viewing $F$ here as an $R$-module so that each $\tau_{i}$ acts as multiplication by $\mu_{i}$. Note, moreover, that the definition of Specht modules carries over unchanged to give modules $S_{\lambda}^{\tau}$ for $H_{d}^{\tau}$ and $\mathcal{S}_{\lambda}^{\tau}$ for $\mathcal{H}_{d}^{\tau}$ for each $\lambda \in \mathcal{M}_{d}(l)$, such that $S_{\lambda}^{\tau} \cong K \otimes_{R} \mathcal{S}_{\lambda}^{\tau}$ and $S_{\lambda}^{\mu} \cong F \otimes_{R} \mathcal{S}_{\lambda}^{\tau}$. The following lemma is well known, but the proof given here is quite instructive.

Lemma 4.1. The algebra $H_{d}^{\tau}$ is split semisimple. Moreover, the Specht modules $S_{\lambda}^{\tau}$ give a complete set of pairwise non-isomorphic irreducible $H_{d}^{\tau}$-modules.

Proof. For each $i=1, \ldots, l$, let $H_{d}^{\tau_{i}}$ denote the degenerate cyclotomic Hecke algebra over $K$ defined by the polynomial $f(x)=\left(x-\tau_{i}\right)$. There is an isomorphism $H_{d}^{\tau_{i}} \stackrel{\sim}{\rightarrow} K S_{d}$ which is the identity on $S_{d}$ and maps $x_{1}$ to $\tau_{i}$. Since $K$ is a field of characteristic zero, we get from this and the classical representation theory of the symmetric group that each $H_{d}^{\tau_{i}}$ is a split semisimple algebra and that the Specht modules $S_{\lambda}^{\tau_{i}}$ for all partitions $\lambda$ of $d$ give a complete set of pairwise non-isomorphic irreducible $H_{d}^{\tau_{i}}$-modules. Since $\tau_{1}, \ldots, \tau_{l}$ are algebraically independent, the proof of [BK3, Corollary 5.20] shows that there is an isomorphism

$$
H_{d}^{\tau} \cong \bigoplus_{d_{1}+\cdots+d_{l}=d} H_{d_{1}}^{\tau_{1}} \otimes \cdots \otimes H_{d_{l}}^{\tau_{l}}
$$

under which $S_{\lambda}^{\tau}$ corresponds to the outer tensor product $S_{\lambda^{(1)}}^{\tau_{1}} \otimes \cdots \otimes S_{\lambda^{(l)}}^{\tau_{l}}$ of Specht modules. The lemma follows.

Lemma4.1 implies that all the Specht modules $\left\{S_{\lambda}^{\tau} \mid \lambda \in X_{d}^{\tau}\right\}$ have different central characters. One can also see this directly by observing from the combinatorial definition that the tuples $\boldsymbol{i}_{\lambda}^{\tau}$ for $\lambda \in \mathcal{M}_{d}(l)$ are in different $\sim$-equivalence classes, i.e. in the generic case the map

$$
\mathcal{M}_{d}(l) \rightarrow X_{d}^{\tau}, \quad \lambda \mapsto \boldsymbol{i}_{\lambda}^{\tau}
$$

is injective. Actually, it is a bijection, by a trivial special case of the following lemma completing the classification of blocks of $H_{d}^{\mu}$ in general.

Lemma 4.2. $X_{d}^{\mu}=\left\{\boldsymbol{i}_{\lambda}^{\mu} \mid \lambda \in \mathcal{M}_{d}(l)\right\}$.

Proof. We have already noted that all $i_{\lambda}^{\mu}$ belong to $X_{d}^{\mu}$. Conversely, we need to show that every maximal ideal of $Z\left(H_{d}^{\mu}\right)$ is of the form $\operatorname{ker} \bar{\chi}\left(i_{\lambda}^{\mu}\right)$ for some $\lambda \in \mathcal{M}_{d}(l)$. We claim that $\prod_{\lambda \in \mathcal{M}_{d}(l)} \operatorname{ker} \bar{\chi}\left(i_{\lambda}^{\mu}\right)=0$. This is more than enough to complete the proof, for if $I$ is any prime ideal of $Z\left(H_{d}^{\mu}\right)$, then the claim implies that $\operatorname{ker} \bar{\chi}\left(\boldsymbol{i}_{\lambda}^{\mu}\right) \subseteq I$ for some $\lambda \in \mathcal{M}_{d}(l)$, hence $I=\operatorname{ker} \bar{\chi}\left(i_{\lambda}^{\mu}\right)$. To prove the claim, note that the following 
diagram commutes:

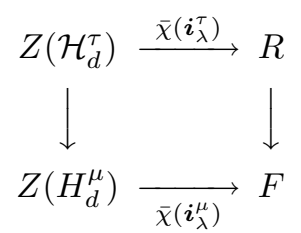

where the vertical maps are defined by evaluating each $\tau_{i}$ at $\mu_{i}$. The left hand map is surjective by Theorem 1 . Hence given $z \in \operatorname{ker} \bar{\chi}\left(i_{\lambda}^{\mu}\right)$ we can find a pre-image $\hat{z} \in Z\left(\mathcal{H}_{d}^{\tau}\right)$ and, replacing $\hat{z}$ by $\hat{z}-\left(\bar{\chi}\left(\boldsymbol{i}_{\lambda}^{\tau}\right)\right)(\hat{z})$ if necessary, we can even assume that $\hat{z} \in \operatorname{ker} \bar{\chi}\left(\boldsymbol{i}_{\lambda}^{\tau}\right)$. This shows that each $\operatorname{ker} \bar{\chi}\left(\boldsymbol{i}_{\lambda}^{\mu}\right)$ is the image of $\operatorname{ker} \bar{\chi}\left(\boldsymbol{i}_{\lambda}^{\tau}\right) \subseteq Z\left(\mathcal{H}_{d}^{\tau}\right)$. Now we have that $\mathcal{H}_{d}^{\tau} \subseteq H_{d}^{\tau}$, and in the semisimple algebra $H_{d}^{\tau}$ it is certainly the case that $\prod_{\lambda \in \mathcal{M}_{d}(l)} \operatorname{ker} \bar{\chi}\left(\boldsymbol{i}_{\lambda}^{\tau}\right)=0$ because the $\bar{\chi}\left(\boldsymbol{i}_{\lambda}^{\tau}\right)$ 's for all $\lambda \in \mathcal{M}_{d}(l)$ are the central characters of a full set of irreducible $H_{d}^{\tau}$-modules, thanks to Lemma4.1.

For $i \in X_{d}^{\mu}$, let $b(\boldsymbol{i})$ be the primitive central idempotent corresponding to the central character $\bar{\chi}(\boldsymbol{i})$, that is, $b(\boldsymbol{i})$ is the unique element of $Z\left(H_{d}^{\mu}\right)$ that acts as one on irreducible modules of central character $\bar{\chi}(\boldsymbol{i})$ and as zero on all other irreducibles. Thus, we have that

$$
H_{d}^{\mu}=\bigoplus_{i \in X_{d}^{\mu}} b(i) H_{d}^{\mu}
$$

This is the decomposition of $H_{d}^{\mu}$ into blocks. Similarly, recalling the bijection $\mathcal{M}_{d}(l) \rightarrow X_{d}^{\tau}, \lambda \mapsto \boldsymbol{i}_{\lambda}^{\tau}$, we can define idempotents $b(\lambda) \in Z\left(H_{d}^{\tau}\right)$ for each $\lambda \in \mathcal{M}_{d}(l)$ such that $b(\lambda)$ acts as one on $S_{\lambda}^{\tau}$ and as zero on all other Specht modules. In view of Lemma 4.1, the resulting decomposition

$$
H_{d}^{\tau}=\bigoplus_{\lambda \in \mathcal{M}_{d}(l)} b(\lambda) H_{d}^{\tau}
$$

is the Wedderburn decomposition of the semisimple algebra $H_{d}^{\tau}$.

Since $R$ is a Noetherian ring complete with respect to the maximal ideal $\mathfrak{m}$, and, moreover, we know that $Z\left(\mathcal{H}_{d}^{\tau}\right)$ surjects onto $Z\left(H_{d}^{\mu}\right)$ by Theorem 1 , there is a unique lift of each $b(\boldsymbol{i}) \in Z\left(H_{d}^{\mu}\right)$ to a central idempotent $\hat{b}(\boldsymbol{i}) \in \mathcal{H}_{d}^{\tau}$; see e.g. [E, Corollary 7.5]. This lifts the block decomposition of $H_{d}^{\mu}$ to a decomposition

$$
H_{d}^{\tau}=\bigoplus_{i \in X_{d}^{\mu}} \hat{b}(i) H_{d}^{\tau}
$$

of the semisimple algebra $H_{d}^{\tau}$. Finally, the commutative diagram from the proof of Lemma 4.2 implies for each $i \in X_{d}^{\mu}$ that

$$
\hat{b}(\boldsymbol{i}) H_{d}^{\tau}=\bigoplus_{\substack{\lambda \in \mathcal{M}_{d}^{(l)} \\ \boldsymbol{i}_{\lambda}^{\sim} \sim \boldsymbol{i}}} b(\lambda) H_{d}^{\tau} .
$$

Now we can prove the only new result of the section, as follows.

Theorem 4.3. For $\boldsymbol{i} \in X_{d}^{\mu}$, the dimension of the center of the block $b(\boldsymbol{i}) H_{d}^{\mu}$ is equal to the number of l-multipartitions $\lambda$ of $d$ such that $\boldsymbol{i}_{\lambda}^{\mu} \sim \boldsymbol{i}$.

Proof. By Theorem $1, Z\left(\mathcal{H}_{d}^{\tau}\right)$ is a free $R$-module of finite rank. Since $R$ is a local ring, it follows that the summand $Z\left(\hat{b}(\boldsymbol{i}) \mathcal{H}_{d}^{\tau}\right)=\hat{b}(\boldsymbol{i}) Z\left(\mathcal{H}_{d}^{\tau}\right)$ is also free, of rank equal to $\operatorname{dim}_{K} Z\left(\hat{b}(\boldsymbol{i}) H_{d}^{\tau}\right)$. Since each $b(\lambda) H_{d}^{\tau}$ is a full matrix algebra with a one dimensional center, we know from the preceeding discussion that the latter dimension is 
equal to the number of $l$-multipartitions $\lambda$ with $\boldsymbol{i}_{\lambda}^{\mu} \sim \boldsymbol{i}$. By Theorem 1 again, the isomorphism $F \otimes_{R} \mathcal{H}_{d}^{\tau} \stackrel{\sim}{\rightarrow} H_{d}^{\mu}$ induces an isomorphism $F \otimes_{R} Z\left(\mathcal{H}_{d}^{\tau}\right) \stackrel{\sim}{\rightarrow} Z\left(H_{d}^{\mu}\right)$. From this, we get an isomorphism $F \otimes_{R} Z\left(\hat{b}(\boldsymbol{i}) \mathcal{H}_{d}^{\tau}\right) \stackrel{\sim}{\rightarrow} Z\left(b(\boldsymbol{i}) H_{d}^{\mu}\right)$. So $\operatorname{dim}_{F} Z\left(b(\boldsymbol{i}) H_{d}^{\mu}\right)$ is the same as the rank of $Z\left(\hat{b}(\boldsymbol{i}) \mathcal{H}_{d}^{\tau}\right)$, i.e. the number of $l$-multipartitions $\lambda$ of $d$ with $i_{\lambda}^{\mu} \sim i$

\section{The Center of Parabolic CATEgory $\mathcal{O}$}

Let $\mathfrak{g}=\mathfrak{g l}_{n}(\mathbb{C})$ with natural module $V$. We denote the standard basis for $V$ by $v_{1}, \ldots, v_{n}$ and use the notation $e_{i, j}$ for the matrix units in $\mathfrak{g}$. Let $\mathfrak{t}$ be the subalgebra of $\mathfrak{g}$ of diagonal matrices and let $\mathfrak{b}$ be the standard Borel subalgebra of upper triangular matrices. Let $\varepsilon_{1}, \ldots, \varepsilon_{n}$ be the basis for $\mathfrak{t}^{*}$ dual to the standard basis $e_{1,1}, \ldots, e_{n, n}$ for $\mathfrak{t}$. We write $L(\alpha)$ for the irreducible highest weight module of highest weight $(\alpha-\rho)$, where $\rho$ is the weight $-\varepsilon_{2}-2 \varepsilon_{3}-\cdots-(n-1) \varepsilon_{n}$. Viewing elements of $S(\mathfrak{t})$ as polynomial functions on $\mathfrak{t}^{*}$, the Harish-Chandra homomorphism

$$
\Psi: Z(\mathfrak{g}) \stackrel{\sim}{\longrightarrow} S(\mathfrak{t})^{S_{n}}
$$

can be defined by declaring that $\Psi(z)$ is the unique element of $S(\mathfrak{t})$ with the property that $z$ acts on $L(\alpha)$ by the scalar $(\Psi(z))(\alpha)$ for each $\alpha \in \mathfrak{t}^{*}$. Its image is the algebra $S(\mathfrak{t})^{S_{n}}$ of symmetric polynomials (for the usual action of $S_{n}$ on $\mathfrak{t}$ not the dot action).

In order to write down a canonical generating set for $Z(\mathfrak{g})$, let $u$ be an indeterminate and set $\tilde{e}_{i, j}:=e_{i, j}+\delta_{i, j}(u+1-i)$. Then the coefficients $z_{1}, \ldots, z_{n}$ of the polynomial

$$
z(u)=\sum_{r=0}^{n} z_{r} u^{n-r}:=\sum_{w \in S_{n}} \operatorname{sgn}(w) \tilde{e}_{w 1,1} \cdots \tilde{e}_{w n, n} \in U(\mathfrak{g})[u]
$$

are algebraically independent generators for the center $Z(\mathfrak{g})$ of $U(\mathfrak{g})$; see e.g. CL, $\S 2.2]$. We adopt the convention that $z_{r}=0$ for $r>n$. The image of $z(u)$ under $\Psi$ (extended in the obvious way to polynomials in the indeterminate $u$ ) is given by the formula

$$
\Psi(z(u))=\left(u+e_{1,1}\right) \cdots\left(u+e_{n, n}\right) .
$$

Hence, for $\alpha=\sum_{i=1}^{n} a_{i} \varepsilon_{i} \in \mathfrak{t}^{*}$, the central element $z_{r}$ acts on $L(\alpha)$ as the scalar $e_{r}(\alpha)=e_{r}\left(a_{1}, \ldots, a_{n}\right)$, the $r$ th elementary symmetric function evaluated at the numbers $a_{1}, \ldots, a_{n}$. Let $P$ denote the free abelian group on the basis $\left\{\gamma_{a} \mid a \in \mathbb{C}\right\}$. For $\alpha=\sum_{i=1}^{n} a_{i} \varepsilon_{i} \in \mathfrak{t}^{*}$, let $\theta(\alpha)=\gamma_{a_{1}}+\cdots+\gamma_{a_{n}} \in P$. The point of this definition is that $L(\alpha)$ and $L(\beta)$ have the same central character if and only if $\theta(\alpha)=\theta(\beta)$. In this way, we have parametrized the central characters of $U(\mathfrak{g})$ by the set of all $\nu \in P$ whose coefficients are non-negative integers summing to $d$.

Let $\Delta: U(\mathfrak{g}) \rightarrow U(\mathfrak{g}) \otimes U(\mathfrak{g})$ be the canonical comultiplication on the universal enveloping algebra of $\mathfrak{g}$. We are only going to need to work with the homomorphism $\delta: U(\mathfrak{g}) \rightarrow U(\mathfrak{g}) \otimes \operatorname{End}_{\mathbb{C}}(V)$ obtained by composing $\Delta$ with the map $1 \otimes \varphi$ where $\varphi$ : $U(\mathfrak{g}) \rightarrow \operatorname{End}_{\mathbb{C}}(V)$ here is the algebra homomorphism arising from the representation of $\mathfrak{g}$ on $V$. Also, let

$$
\Omega=\sum_{i, j=1}^{n} e_{i, j} \otimes e_{j, i} \in U(\mathfrak{g}) \otimes \operatorname{End}_{\mathbb{C}}(V) .
$$

The following lemma is probably classical. 
Lemma 5.1. For $r \geq 0$, we have that

$$
\delta\left(z_{r}\right)=z_{r} \otimes 1+\sum_{s=0}^{r-1}(-1)^{s}\left(z_{r-1-s} \otimes 1\right) \Omega^{s} .
$$

Proof. Both sides of the equation are elements of $U(\mathfrak{g}) \otimes \operatorname{End}_{\mathbb{C}}(V)$, so can be viewed as $n \times n$ matrices with entries in $U(\mathfrak{g})$. To see that these matrices are equal, it suffices to check that their entries act in the same way on sufficiently many finite dimensional irreducible representations of $\mathfrak{g}$. This reduces to the following problem. Take $\alpha=\sum_{i=1}^{n} a_{i} \varepsilon_{i} \in \mathfrak{t}^{*}$ such that $L(\alpha)$ is finite dimensional and

$$
L(\alpha) \otimes V \cong \bigoplus_{i=1}^{n} L\left(\alpha+\varepsilon_{i}\right)
$$

We need to show that the left and right hand sides of the given equation define the same endomorphism of $L(\alpha) \otimes V$. For such an $\alpha$, let $M:=L(\alpha) \otimes V$ and write $v_{+}$for a highest weight vector in $L(\alpha)$. For $i=0, \ldots, n$, define $M_{i}$ to be the submodule of $M$ generated by the vectors $v_{+} \otimes v_{j}(j \leq i)$. Since $v_{+} \otimes v_{i}$ is a highest weight vector of weight $\alpha+\varepsilon_{i}$ modulo $M_{i-1}$, the assumption on $\alpha$ implies that $0=M_{0} \subset M_{1} \subset \cdots \subset M_{n}=M$ is a filtration of $M$ such that $M_{i} / M_{i-1} \cong L\left(\alpha+\varepsilon_{i}\right)$. Since the filtration splits, there is a unique highest weight vector $x_{i} \in M$ such that $x_{i} \equiv v_{+} \otimes v_{i}\left(\bmod M_{i-1}\right)$. Now we just check that the left and right hand sides of the given equation act on these highest weight vectors by the same scalar for each $i=1, \ldots, n$. Of course $\delta\left(z_{r}\right)$ acts on $x_{i}$ as $e_{r}\left(\alpha+\varepsilon_{i}\right)$, while each $z_{t} \otimes 1$ acts as $e_{t}(\alpha)$ on all of $M$. Finally, since $\Omega$ defines a $\mathfrak{g}$-module endomorphism of $M$, it leaves $M_{i-1}$ invariant and maps $x_{i}$ to a scalar multiple of itself. To compute the scalar, note that

$$
\begin{aligned}
\Omega\left(v_{+} \otimes v_{i}\right) & =\sum_{j \leq i}\left(e_{i, j} v_{+}\right) \otimes v_{j}=\left(e_{i, i} v_{+}\right) \otimes v_{i}+\sum_{j<i}\left(e_{i, j} v_{+}\right) \otimes v_{j} \\
& =\left(a_{i}+i-1\right) v_{+} \otimes v_{i}+\sum_{j<i}\left(e_{i, j}\left(v_{+} \otimes v_{j}\right)-v_{+} \otimes v_{i}\right) \\
& \equiv a_{i} v_{+} \otimes v_{i} \quad\left(\bmod M_{i-1}\right) .
\end{aligned}
$$

Hence, $\Omega x_{i}=a_{i} x_{i}$. So the equation we are trying to prove reduces to checking that

$$
e_{r}\left(\alpha+\varepsilon_{i}\right)=e_{r}(\alpha)+\sum_{s=0}^{r-1}(-1)^{s} e_{r-1-s}(\alpha) a_{i}^{s}
$$

for each $i=1, \ldots, n$. This follows from the following general identity which is true for all $r, k \geq 0$ :

$$
e_{r}\left(u_{1}, \ldots, u_{k}, u+1\right)=e_{r}\left(u_{1}, \ldots, u_{k}, u\right)+\sum_{s=0}^{r-1}(-1)^{s} e_{r-1-s}\left(1, \ldots, u_{k}, u\right) u^{s} .
$$

To see this, expand both sides using the obvious formula

$$
e_{t}\left(u_{1}, \ldots, u_{k}, v\right)=e_{t}\left(u_{1}, \ldots, u_{k}\right)+e_{t-1}\left(u_{1}, \ldots, u_{k}\right) v .
$$

Let $M$ be any $\mathfrak{g}$-module. Recall from $[\mathrm{AS}, \S 2.2]$ that the degenerate affine Hecke algebra $H_{d}$ over the ground field $\mathbb{C}$ acts naturally on the right on $M \otimes V^{\otimes d}$ by $\mathfrak{g}$ module endomorphisms. The action of each $w \in S_{d}$ arises from its usual action on $V^{\otimes d}$ by place permutation. The action of $x_{1}$ (from which one can deduce the action 
of all other $x_{i}$ 's) is the same as left multiplication by $\Omega \otimes 1^{\otimes(d-1)}$. For any partition $\mu$ with $\ell(\mu) \leq d$, recall the notation $p_{d}(\mu)$ introduced just before Theorem 3.2 . we are now viewing this expression as an element of $H_{d}$.

Lemma 5.2. For any $r, d \geq 0$ and any highest weight module $M$ of highest weight $\alpha-\rho \in \mathfrak{t}^{*}$, the endomorphism of $M \otimes V^{\otimes d}$ defined by left multiplication by $z_{r} \in Z(\mathfrak{g})$ is equal to the endomorphism defined by right multiplication by

$$
\sum_{\mu}(-1)^{|\bar{\mu}|}\left(\begin{array}{l}
d-\ell(\bar{\mu}) \\
d-\ell(\mu)
\end{array}\right) e_{r-|\mu|}(\alpha) p_{d}(\bar{\mu}) \in Z\left(H_{d}\right)
$$

where the sum is over partitions $\mu$ of length $\ell(\mu) \leq d$ and size $|\mu| \leq r$, and $\bar{\mu}$ denotes the partition $\left(\mu_{1}-1 \geq \cdots \geq \mu_{\ell(\mu)}-1\right)$ obtained from $\mu$ by removing the first column of its diagram.

Proof. Let $\delta_{d}: U(\mathfrak{g}) \rightarrow U(\mathfrak{g}) \otimes \operatorname{End}_{\mathbb{C}}(V)^{\otimes d}$ be the map defined inductively by setting $\delta_{0}=1$ and $\delta_{d}=\left(\delta \otimes 1^{\otimes(d-1)}\right) \circ \delta_{d-1}$ for $d \geq 1$. Let $\Omega_{i}:=\left(\delta_{i-1} \otimes 1\right)(\Omega) \otimes 1^{(d-i)} \in$ $U(\mathfrak{g}) \otimes \operatorname{End}_{\mathbb{C}}(V)^{\otimes d}$. If we adopt the convention that $(-\Omega)^{-1}=1$, we can write the conclusion of Lemma 5.1 simply as

$$
\delta\left(z_{r}\right)=\sum_{s=0}^{r}\left(z_{r-s} \otimes 1\right)(-\Omega)^{s-1} .
$$

Proceeding from this by induction on $d$, it is straightforward to deduce that

$$
\delta_{d}\left(z_{r}\right)=\sum_{\substack{s_{1}, \ldots, s_{d} \geq 0 \\ s_{1}+\cdots+s_{d} \leq r}}\left(z_{r-s_{1}-\cdots-s_{d}} \otimes 1^{\otimes d}\right)\left(-\Omega_{1}\right)^{s_{1}-1} \cdots\left(-\Omega_{d}\right)^{s_{d}-1}
$$

interpreting the right hand side with the same convention. Since $x_{i+1}=s_{i} x_{i} s_{i}+s_{i}$ and $x_{1}$ acts as $\Omega_{1}$ by definition, one checks by induction that $x_{i}$ acts as $\Omega_{i}$ for each $i$. Hence on applying our expression to $M \otimes V^{\otimes d}$, we deduce that $z_{r}$ acts in the same way as

$$
\sum_{\substack{s_{1}, \ldots, s_{d} \geq 0 \\ s_{1}+\cdots+s_{d} \leq r}} e_{r-s_{1}-\cdots-s_{d}}(\alpha)\left(-x_{1}\right)^{s_{1}-1} \cdots\left(-x_{d}\right)^{s_{d}-1},
$$

again interpreting $\left(-x_{i}\right)^{-1}$ as 1 . It is now a combinatorial exercise to rewrite this expression as formulated in the statement of the lemma.

Corollary 5.3. For any highest weight module $M$, the subalgebra of the algebra $\operatorname{End}_{\mathbb{C}}\left(M \otimes V^{\otimes d}\right)^{\text {op }}$ generated by the endomorphisms $z_{r}(1 \leq r \leq n)$ coincides with the subalgebra generated by $x_{1}^{r}+\cdots+x_{d}^{r}(1 \leq r \leq d)$.

Proof. Since we are working over a field of characteristic 0 , any symmetric polynomial in $x_{1}, \ldots, x_{d}$ lies in the subalgebra generated by the power sums $x_{1}^{r}+\cdots+$ $x_{d}^{r}(1 \leq r \leq d)$. By Lemma 5.2, the endomorphism defined by $z_{r}$ can be expressed as a symmetric polynomial in $x_{1}, \ldots, x_{d}$, so it lies in the subalgebra generated by the power sums.

Conversely, we show by induction on $r \geq 0$ that every homogeneous symmetric polynomial in $x_{1}, \ldots, x_{d}$ of degree $r$ acts on $M \otimes V^{\otimes d}$ in the same way as some element of the subalgebra generated by $z_{1}, \ldots, z_{r+1}$. For the induction step, every homogeneous symmetric polynomial of degree $r$ lies in the subalgebra generated by the power sums $x_{1}^{s}+\cdots+x_{d}^{s}(1 \leq s \leq r)$. By induction all of these power sums with $s<r$ certainly lie in the subalgebra generated by $z_{1}, \ldots, z_{r+1}$, so it just 
remains to show that $x_{1}^{r}+\cdots+x_{d}^{r}$ does too. By Lemma 5.2, the image of $z_{r+1}$ is the same as the image of $x_{1}^{r}+\cdots+x_{d}^{r}$ (which is the term $p_{d}(\bar{\mu})$ when $\mu=(r+1)$ ) plus a linear combination of symmetric polynomials in $x_{1}, \ldots, x_{d}$ of strictly smaller degree, which we already have by the induction hypothesis.

Let $\mu=\left(\mu_{1}, \ldots, \mu_{l}\right)$ be a composition of $n$ and let $\mathfrak{p}$ be the corresponding standard parabolic subalgebra of $\mathfrak{g}$ with standard Levi subalgebra $\mathfrak{h} \cong \mathfrak{g l}_{\mu_{1}}(\mathbb{C}) \oplus$ $\cdots \oplus \mathfrak{g l}_{\mu_{l}}(\mathbb{C})$, as in the introduction. We are interested in the category $\mathcal{O}^{\mu}$ of all finitely generated $\mathfrak{g}$-modules that are locally finite over $\mathfrak{p}$ and integrable over $\mathfrak{h}$. Let $H_{d}^{\mu}$ denote the degenerate cyclotomic Hecke algebra associated to the polynomial $\left(x-\mu_{1}\right) \cdots\left(x-\mu_{l}\right)$ from the previous section over the ground field $F=\mathbb{C}$. We are going to apply the Schur-Weyl duality for higher levels from BK3 (taking the choice of origin there to be $\boldsymbol{c}=(n, \ldots, n))$ to connect the category $\mathcal{O}^{\mu}$ to the finite dimensional algebras $H_{d}^{\mu}$ for all $d \geq 0$. Actually, BK3] only considered the special case that $\mu$ is a partition, i.e. $\mu_{1} \geq \cdots \geq \mu_{l}$, so we will need to extend some of the results of [BK3] to the general case as we go.

To start with, we need some combinatorial definitions. Let

$$
\mathrm{Col}^{\mu}=\left\{\begin{array}{l|l}
\alpha=\sum_{i=1}^{n} a_{i} \varepsilon_{i} \in \mathfrak{t}^{*} & \begin{array}{c}
a_{1}, \ldots, a_{n} \in \mathbb{Z} \text { such that } a_{i}>a_{i+1} \\
\text { for all } i \neq \mu_{1}, \mu_{1}+\mu_{2}, \ldots, \mu_{1}+\cdots+\mu_{l}
\end{array}
\end{array}\right\},
$$

so called because its elements can be visualized as column strict tableaux of column shape $\mu$ as in [B1, §2] or [BK3, §4]. The irreducible modules in $\mathcal{O}^{\mu}$ are the modules $\left\{L(\alpha) \mid \alpha \in \mathrm{Col}^{\mu}\right\}$. Hence the set $Y^{\mu}=\left\{\theta(\alpha) \mid \alpha \in \mathrm{Col}^{\mu}\right\}$ naturally parametrizes the central characters arising from modules in $\mathcal{O}^{\mu}$. Given $\nu \in Y^{\mu}$, we let $\operatorname{Col}_{\nu}^{\mu}=$ $\left\{\alpha \in \mathrm{Col}^{\mu} \mid \theta(\alpha)=\nu\right\}$ and define $\mathcal{O}_{\nu}^{\mu}$ to be the Serre subcategory of $\mathcal{O}^{\mu}$ generated by the modules $\left\{L(\alpha) \mid \alpha \in \mathrm{Col}_{\nu}^{\mu}\right\}$. The category $\mathcal{O}^{\mu}$ then decomposes as

$$
\mathcal{O}^{\mu}=\bigoplus_{\nu \in Y^{\mu}} \mathcal{O}_{\nu}^{\mu} .
$$

This is the same as the central character decomposition of $\mathcal{O}^{\mu}$ that was described in the introduction.

Let $\gamma \in Y^{\mu}$ be the special element $\gamma=\sum_{i=1}^{l} \sum_{a=1}^{\mu_{i}} \gamma_{a}$. The key feature of $\gamma$ is that the set $\operatorname{Col}_{\gamma}^{\mu}$ contains just one weight $\alpha$. In other words, for this $\alpha, L(\alpha)$ is the unique irreducible module in $\mathcal{O}^{\mu}$ with central character parametrized by $\gamma$. This special irreducible module, which we denote henceforth by $P^{\mu}$, is automatically projective.

For $d \geq 0$, let

$$
\begin{aligned}
Y_{d}^{\mu} & =\left\{\gamma-\left(\gamma_{i_{1}}-\gamma_{i_{1}+1}\right)-\cdots-\left(\gamma_{i_{d}}-\gamma_{i_{d}+1}\right) \in Y^{\mu} \mid i_{1}, \ldots, i_{d} \in \mathbb{Z}\right\}, \\
\mathrm{Col}_{d}^{\mu} & =\left\{\alpha \in \mathrm{Col}^{\mu} \mid \theta(\alpha) \in Y_{d}^{\mu}\right\} .
\end{aligned}
$$

The irreducible modules $\left\{L(\alpha) \mid \alpha \in \mathrm{Col}_{d}^{\mu}\right\}$ are significant because they are exactly the irreducible constituents of the module $P^{\mu} \otimes V^{\otimes d}$. This statement is proved in BK3, Lemma 4.2] in the case that $\mu$ is a partition, and the same argument works in general.

Lemma 5.4. The map $Y_{d}^{\mu} \rightarrow X_{d}^{\mu}$ sending $\nu=\gamma-\left(\gamma_{i_{1}}-\gamma_{i_{1}+1}\right)-\cdots-\left(\gamma_{i_{d}}-\gamma_{i_{d}+1}\right)$ to $\boldsymbol{i}=\left(i_{1}, \ldots, i_{d}\right)$ is injective with image equal to

$$
\left\{\boldsymbol{i}_{\lambda}^{\mu} \mid \lambda \in \mathcal{M}_{d}(l) \text { such that } \ell\left(\lambda^{(r)}\right) \leq \mu_{r} \text { for } r=1, \ldots, l\right\} .
$$


For $\nu \in Y_{d}^{\mu}$ corresponding to $i \in X_{d}^{\mu}$ in this way, the map $\mathrm{Col}_{\nu}^{\mu} \rightarrow \mathcal{M}_{d}(l)$ sending $\alpha=\sum_{i=1}^{n} a_{i} \varepsilon_{i}$ to the multipartition $\lambda=\left(\lambda^{(1)}, \ldots, \lambda^{(l)}\right)$ such that $\lambda^{(r)}=$ $\left(a_{\mu_{1}+\cdots+\mu_{r-1}+1}-\mu_{r}, a_{\mu_{1}+\cdots+\mu_{r-1}+2}-\left(\mu_{r}-1\right), \ldots, a_{\mu_{1}+\cdots+\mu_{r-1}+\mu_{r}}-1\right)$ is injective with image equal to $\left\{\lambda \in \mathcal{M}_{d}(l) \mid i_{\lambda}^{\mu} \sim \boldsymbol{i}\right\}$.

Proof. We leave this as a combinatorial exercise. It is helpful to use the interpretation of $\mathrm{Col}_{\nu}^{\mu}$ as the set of column strict tableaux of column shape $\mu$ and type $\nu$ as in [BK3, §4].

As explained before Lemma 5.2, the degenerate affine Hecke algebra $H_{d}$ acts on the right on $P^{\mu} \otimes V^{\otimes d}$ by $\mathfrak{g}$-module endomorphisms. We let

$$
\rho^{\mu}: H_{d} \rightarrow \operatorname{End}_{\mathfrak{g}}\left(P^{\mu} \otimes V^{\otimes d}\right)^{\text {op }}
$$

be the resulting algebra homomorphism. The following lemma is the key to extending the results from BK3 to general $\mu$. The proof of this is non-trivial: the parabolic categories $\mathcal{O}^{\mu}$ and $\mathcal{O}^{\mu^{\prime}}$ for conjugate compositions $\mu$ and $\mu^{\prime}$ are not in general equivalent so it is necessary to work at the level of derived categories adapting an argument due to Mazorchuk and Stroppel.

Lemma 5.5. Given another composition $\mu^{\prime} \sim \mu$, there is an algebra isomorphism $\iota_{\mu, \mu^{\prime}}$ making the following diagram commute:

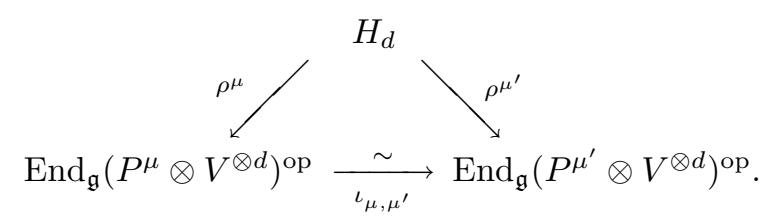

Moreover, $\iota_{\mu, \mu^{\prime}}$ intertwines the natural actions of $Z(\mathfrak{g})$ on the two endomorphism algebras.

Proof. As explained in the proof of [MS, Theorem 5.4], there is an adjoint pair $(F, G)$ of functors (which are compositions of certain derived Zuckerman functors) between the bounded derived categories,

with the following properties:

$$
D^{b}\left(\mathcal{O}^{\mu}\right) \underset{G}{\stackrel{F}{\rightleftarrows}} D^{b}\left(\mathcal{O}^{\mu^{\prime}}\right),
$$

(i) $F$ and $G$ commute with tensoring with finite dimensional $\mathfrak{g}$-modules; that is, for any finite dimensional $\mathfrak{g}$-module $V$ there are given natural isomorphisms $\alpha_{V}: F \circ ? \otimes V \rightarrow ? \otimes V \circ F$ and $\beta_{V}: G \circ ? \otimes V \rightarrow ? \otimes V \circ G$ such that the following two diagrams commute for any morphism $f: V \rightarrow W$ of finite dimensional $\mathfrak{g}$-modules:

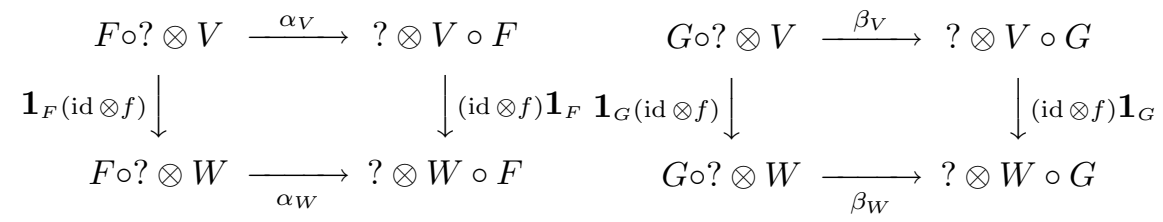

(ii) The isomorphisms $\alpha_{V}$ and $\beta_{V}$ are compatible with the unit $\eta: \operatorname{Id} \rightarrow G \circ F$ and counit $\varepsilon: F \circ G \rightarrow$ Id of the canonical adjunction between $F$ and $G$, i.e. the following diagrams commute: 


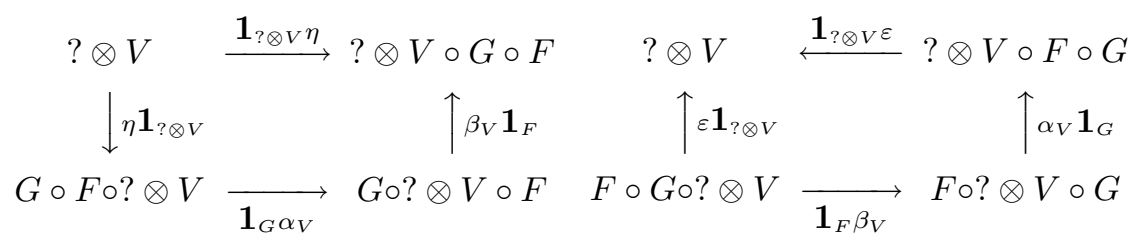

(iii) $F$ and $G$ restrict to mutually inverse equivalences of categories between $\mathcal{O}_{\gamma}^{\mu}$ and $\mathcal{O}_{\gamma}^{\mu^{\prime}}$.

(iv) The following associativity pentagon commutes for any two finite dimensional $\mathfrak{g}$-modules $V$ and $W$ :

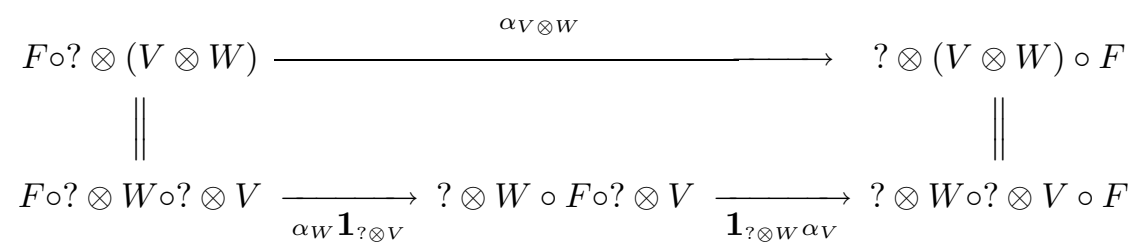

(v) $F$ transforms the endomorphism of an object $M$ defined by left multiplication by $z \in Z(\mathfrak{g})$ to the endomorphism of $F M$ defined by left multiplication by the same element $z$.

We now explain how to deduce the lemma from these facts. By (iii), we can choose an isomorphism $\varphi_{0}: F\left(P^{\mu}\right) \stackrel{\sim}{\rightarrow} P^{\mu^{\prime}}$. Then define a homomorphism $\varphi_{d}:$ $F\left(P^{\mu} \otimes V^{\otimes d}\right) \rightarrow P^{\mu^{\prime}} \otimes V^{\otimes d}$ by $\varphi_{d}:=\left(\varphi_{0} \otimes \mathrm{id}_{V \otimes d}\right) \circ \alpha_{V^{\otimes d}}$. This is an isomorphism in $D^{b}\left(\mathcal{O}^{\mu^{\prime}}\right)$ by (i). Define another homomorphism $\bar{\varphi}_{d}: P^{\mu} \otimes V^{\otimes d} \rightarrow G\left(P^{\mu^{\prime}} \otimes V^{\otimes d}\right)$ by $\bar{\varphi}_{d}:=G \varphi_{d} \circ \eta_{P^{\mu} \otimes V \otimes d}$. This is an isomorphism in $D^{b}\left(\mathcal{O}^{\mu}\right)$ by (ii). Then we get an algebra homomorphism

$$
\iota_{\mu, \mu^{\prime}}: \operatorname{End}_{\mathfrak{g}}\left(P^{\mu} \otimes V^{\otimes d}\right)^{\mathrm{op}} \rightarrow \operatorname{End}_{\mathfrak{g}}\left(P^{\mu^{\prime}} \otimes V^{\otimes d}\right)^{\mathrm{op}}
$$

mapping $f$ to $\varphi_{d} \circ F f \circ \varphi_{d}^{-1}$ and another algebra homomorphism

$$
\iota_{\mu^{\prime}, \mu}: \operatorname{End}_{\mathfrak{g}}\left(P^{\mu^{\prime}} \otimes V^{\otimes d}\right)^{\mathrm{op}} \rightarrow \operatorname{End}_{\mathfrak{g}}\left(P^{\mu} \otimes V^{\otimes d}\right)^{\mathrm{op}}
$$

sending $g$ to $\bar{\varphi}_{d}^{-1} \circ G g \circ \bar{\varphi}_{d}$, such that $\iota_{\mu, \mu^{\prime}}$ and $\iota_{\mu^{\prime}, \mu}$ are mutual inverses. Moreover, by (v), both homomorphisms intertwine the natural actions of $Z(\mathfrak{g})$. It just remains to check that $\iota_{\mu, \mu^{\prime}}$ is compatible with the action of $H_{d}$. The compatibility of $\iota_{\mu, \mu^{\prime}}$ with the action of each $w \in S_{d}$ follows immediately from the naturality in (i). So it suffices to show that it is compatible with the action of $x_{1}$. For this, we first reduce using (iv) to check compatiblity just in the special case $d=1$. In that case it follows from (v) since by Lemma 5.1 we have that $x_{1}$ acts as left multiplication by $\Omega=z_{2} \otimes 1+z_{1} \otimes 1-\delta\left(z_{2}\right)$, and $z_{2} \otimes 1$ and $z_{1} \otimes 1$ act by the same scalars on $P^{\mu} \otimes V$ and $P^{\mu^{\prime}} \otimes V$.

We can formulate the critical result needed from BK3] as follows. Recall the primitive central idempotents $b(\boldsymbol{i}) \in H_{d}^{\mu}$ for each $\boldsymbol{i} \in X_{d}^{\mu}$ introduced just after Lemma 4.2

Theorem 5.6. The image of $\rho^{\mu}: H_{d} \rightarrow \operatorname{End}_{\mathbb{C}}\left(P^{\mu} \otimes V^{\otimes d}\right)^{\mathrm{op}}$ coincides with the endomorphism algebra $\operatorname{End}_{\mathfrak{g}}\left(P^{\mu} \otimes V^{\otimes d}\right)^{\mathrm{op}}$. Moreover, the representation $\rho^{\mu}$ factors through the quotient $H_{d}^{\mu}$ of $H_{d}$, and the kernel of the induced map $H_{d}^{\mu} \rightarrow \operatorname{End}_{\mathfrak{g}}\left(P^{\mu} \otimes\right.$ 
$\left.V^{\otimes d}\right)^{\mathrm{op}}$ is generated by $(1-e)$, where $e \in H_{d}^{\mu}$ is the central idempotent $e=\sum_{\boldsymbol{i}} b(\boldsymbol{i})$ summing over all $\boldsymbol{i}$ lying in the set

$$
\left\{\boldsymbol{i}_{\lambda}^{\mu} \mid \lambda \in \mathcal{M}_{d}(l) \text { such that } \ell\left(\lambda^{(r)}\right) \leq \mu_{r} \text { for } r=1, \ldots, l\right\}
$$

from Lemma [5.4. Hence, $\rho^{\mu}$ induces an isomorphism between the sum of blocks $e H_{d}^{\mu}$ of $H_{d}^{\mu}$ and the endomorphism algebra $\operatorname{End}_{\mathfrak{g}}\left(P^{\mu} \otimes V^{\otimes d}\right)^{\mathrm{op}}$.

Proof. If $\mu$ is a partition, this follows by [BK3, Theorem 5.13] and BK3, Corollary 6.7]. It then follows for arbitrary $\mu$ too by Lemma 5.5.

Corollary 5.7. The center of $\operatorname{End}_{\mathfrak{g}}\left(P^{\mu} \otimes V^{\otimes d}\right)^{\text {op }}$ is generated by the endomorphisms $z_{1}, \ldots, z_{n}$.

Proof. By Corollary 3.3 we know already that $Z\left(H_{d}^{\mu}\right)$ is generated by the power sums $x_{1}^{r}+\cdots+x_{d}^{r}$ for $1 \leq r \leq d$. By Theorem 5.6] we can identify the endomorphism algebra $\operatorname{End}_{\mathfrak{g}}\left(P^{\mu} \otimes V^{\otimes d}\right)^{\text {op }}$ with $e H_{d}^{\mu}$ for some central idempotent $e \in H_{d}^{\mu}$. So its center is generated by the restrictions of these power sums to the module $P^{\mu} \otimes V^{\otimes d}$. Now apply Corollary 5.3 .

Now let us restrict attention to a single central character. For the rest of the article, we fix $\nu \in Y_{d}^{\mu}$ and define $\boldsymbol{i}=\left(i_{1}, \ldots, i_{d}\right) \in X_{d}^{\mu}$ from $\nu=\gamma-\left(\gamma_{i_{1}}-\gamma_{i_{1}+1}\right)-$ $\cdots-\left(\gamma_{i_{d}}-\gamma_{i_{d}+1}\right)$ as in Lemma 5.4. Let

$$
e_{\nu}^{\mu} \in \operatorname{End}_{\mathfrak{g}}\left(P^{\mu} \otimes V^{\otimes d}\right)^{\mathrm{op}}
$$

be the central idempotent projecting $P^{\mu} \otimes V^{\otimes d}$ onto its component of generalized central character parametrized by $\nu$. Note $\left(P^{\mu} \otimes V^{\otimes d}\right) e_{\nu}^{\mu}$ is non-zero; see e.g. BK3, Lemma 4.2]. Identifying $\operatorname{End}_{\mathfrak{g}}\left(P^{\mu} \otimes V^{\otimes d}\right)^{\mathrm{op}}$ with $e H_{d}^{\mu}$ according to Theorem [5.6, it follows that $e_{\nu}^{\mu}$ is identified with a non-zero central idempotent in $H_{d}^{\mu}$.

Lemma 5.8. $e_{\nu}^{\mu}=b(\boldsymbol{i})$.

Proof. We first prove this in the special case that $\mu$ is a partition. Certainly $e_{\nu}^{\mu}$ is a non-zero sum of the primitive central idempotents $b(\boldsymbol{i})$ for $\boldsymbol{i} \in X_{d}^{\mu}$. So we just need to show that $e_{\nu}^{\mu}$ acts as zero on $S_{\lambda}^{\mu}$ for all $\lambda \in \mathcal{M}_{d}(l)$ with $\boldsymbol{i}_{\lambda}^{\mu} \nsim \boldsymbol{i}$. Given such a $\lambda$, this is clear from the definition of the idempotent $e$ in Theorem 5.6 unless $\ell\left(\lambda^{(r)}\right) \leq \mu_{r}$ for each $r=1, \ldots, l$. In that case, there is a unique weight $\alpha \in \mathrm{Col}_{d}^{\mu}$ mapping to $\lambda$ under the second bijection from Lemma 5.4 and the assumption that $\boldsymbol{i}_{\lambda}^{\mu} \nsim \boldsymbol{i}$ is equivalent to the statement that $\theta(\alpha) \neq \nu$. Let $M(\alpha)$ be the parabolic Verma module in $\mathcal{O}^{\mu}$ of highest weight $(\alpha-\rho)$. By [BK3, Theorem 6.12], we have that

$$
\operatorname{Hom}_{\mathfrak{g}}\left(P^{\mu} \otimes V^{\otimes d}, M(\alpha)\right) \cong S_{\lambda}^{\mu}
$$

as $H_{d}^{\mu}$-modules. Since $M(\alpha)$ and $\left(P^{\mu} \otimes V^{\otimes d}\right) e_{\nu}^{\mu}$ have different generalized central characters, we have that

$$
e_{\nu}^{\mu} \operatorname{Hom}_{\mathfrak{g}}\left(P^{\mu} \otimes V^{\otimes d}, M(\alpha)\right)=\operatorname{Hom}_{\mathfrak{g}}\left(\left(P^{\mu} \otimes V^{\otimes d}\right) e_{\nu}^{\mu}, M(\alpha)\right)=0 .
$$

Hence $e_{\nu}^{\mu} S_{\lambda}^{\mu}=0$ as required.

To deduce the general case, assume still that $\mu$ is a partition and take another composition $\mu^{\prime} \sim \mu$. We can find a central element $z_{\nu} \in Z(\mathfrak{g})$ that acts on $P^{\mu} \otimes V^{\otimes d}$ in the same way as $e_{\nu}^{\mu}$ and on $P^{\mu^{\prime}} \otimes V^{\otimes d}$ in the same way as $e_{\nu}^{\mu^{\prime}}$. In the notation of Lemma 5.5. we have shown that $\rho^{\mu}(b(\boldsymbol{i}))$ coincides with the endomorphism of $P^{\mu} \otimes V^{\otimes d}$ defined by left multiplication by $z_{\nu}$. We need to prove that $\rho^{\mu^{\prime}}(b(i))$ does too. This follows because the map $\iota_{\mu, \mu^{\prime}}$ commutes with the action of $z_{\nu}$. 
Now we can deduce the following refinement of Theorem 5.6.

Theorem 5.9. The map $\rho_{\nu}^{\mu}: b(\boldsymbol{i}) H_{d}^{\mu} \rightarrow \operatorname{End}_{\mathfrak{g}}\left(\left(P^{\mu} \otimes V^{\otimes d}\right) e_{\nu}^{\mu}\right)^{\mathrm{op}}$ induced by the right action of $H_{d}^{\mu}$ on $P^{\mu} \otimes V^{\otimes d}$ is an algebra isomorphism. Moreover, the center of this algebra is generated by the endomorphisms $z_{1}, \ldots, z_{n}$, and is of dimension equal to the number of isomorphism classes of irreducible modules in $\mathcal{O}_{\nu}^{\mu}$.

Proof. The first statement is immediate from Theorem [5.6 since $e_{\nu}^{\mu}=b(\boldsymbol{i})$. The fact that the center of $\operatorname{End}_{\mathfrak{g}}\left(\left(P^{\mu} \otimes V^{\otimes d}\right) e_{\nu}^{\mu}\right)^{\text {op }}$ is generated by the endomorphisms $z_{1}, \ldots, z_{n}$ is immediate from Corollary [5.7. The center is isomorphic to $Z\left(b(\boldsymbol{i}) H_{d}^{\mu}\right)$, which by Theorem 4.3 is of dimension equal to the size of the set $\left\{\lambda \in \mathcal{M}_{d}(l) \mid \boldsymbol{i}_{\lambda}^{\mu} \sim\right.$ $i$ \}. By Lemma 5.4, this is the same as the size of the set $\mathrm{Col}_{\nu}^{\mu}$, that is, the number of isomorphism classes of irreducible modules in $\mathcal{O}_{\nu}^{\mu}$.

We need just one more fact, which is a variation on a result of Irving [I]

Lemma 5.10. The injective hull of any module in $\mathcal{O}_{\nu}^{\mu}$ with a parabolic Verma flag is a finite direct sum of direct summands of $\left(P^{\mu} \otimes V^{\otimes d}\right) e_{\nu}^{\mu}$.

Proof. We claim that every irreducible submodule of a parabolic Verma module in $\mathcal{O}_{\nu}^{\mu}$ embeds into $\left(P^{\mu} \otimes V^{\otimes d}\right) e_{\nu}^{\mu}$. Since $\left(P^{\mu} \otimes V^{\otimes d}\right) e_{\nu}^{\mu}$ is injective this implies the lemma. To prove the claim, recall that $\nu$ is an element of the set $Y_{d}^{\mu}$ that parametrizes the central characters arising from irreducible constituents of $P^{\mu} \otimes$ $V^{\otimes d}$. So the claim follows by [BK3, Theorem 4.8] and [BK3, Corollary 4.6] in the special case that $\mu$ is actually a partition. Essentially the same proof as there proves the analogue of this theorem for arbitrary $\mu$, providing one replaces the definition of "standard tableau" used in BK3 with the less familiar combinatorial notion from B1, (2.2)].

Now finally we consider the commutative diagram

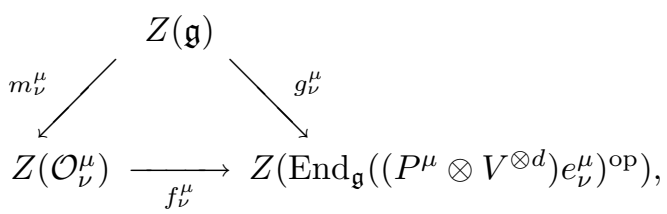

where $m_{\nu}^{\mu}, f_{\nu}^{\mu}$ and $g_{\nu}^{\mu}$ are the natural multiplication maps.

Theorem 5.11. In the above diagram, the maps $m_{\nu}^{\mu}$ and $g_{\nu}^{\mu}$ are surjective and the map $f_{\nu}^{\mu}$ is an isomorphism. Hence, $Z\left(\mathcal{O}_{\nu}^{\mu}\right)$ is isomorphic to $Z\left(b(i) H_{d}^{\mu}\right)$ and is of dimension equal to the number of isomorphism classes of irreducible module in $\mathcal{O}_{\nu}^{\mu}$.

Proof. We first prove that $f_{\nu}^{\mu}$ is injective. Suppose we are given a natural transformation $z \in Z\left(\mathcal{O}_{\nu}^{\mu}\right)$ defining the zero endomorphism of $\left(P^{\mu} \otimes V^{\otimes d}\right) e_{\nu}^{\mu}$. To show that $z=0$, we need to show that $z$ defines the zero endomorphism of every module $M \in \mathcal{O}_{\nu}^{\mu}$. Let $P$ be the projective cover of $M$ and $I$ the injective hull of $P$. Since $P$ has a parabolic Verma flag by general theory, Lemma 5.10 implies that $I$ is a finite direct sum of summands of $\left(P^{\mu} \otimes V^{\otimes d}\right) e_{\nu}^{\mu}$. Hence $z$ defines the zero endomorphism of $I$. Since $P$ embeds into $I$ and surjects onto $M$, we get from this that $z$ defines the zero endomorphism of $M$ too. Now to finish the proof of the theorem, we know already from Theorem 5.9 that $g_{\nu}^{\mu}$ is surjective. Hence by the commutativity of the diagram, $m_{\nu}^{\mu}$ and $f_{\nu}^{\mu}$ must both be surjective too. The remaining statements are immediate from Theorem 5.9. 
Finally, we note for any $\nu \in Y^{\mu}$ that tensoring with a sufficiently large power of determinant induces an equivalence between $\mathcal{O}_{\nu}^{\mu}$ and $\mathcal{O}_{\nu^{\prime}}^{\mu}$ for some $\nu^{\prime} \in Y_{d}^{\mu}$ and some $d \geq 0$. Given this, Theorem 2 from the introduction follows from Theorem [5.11.

\section{ACKNOWLEDGEMENTS}

Thanks go to Brian Boe, Alexander Kleshchev and Victor Ostrik for helpful discussions, Iain Gordon for pointing out Müller's theorem, and Jim Humphreys for the reference to Jantzen's paper mentioned above.

\section{REFERENCES}

[AS] T. Arakawa and T. Suzuki, Duality between $\mathfrak{s l}_{n}(\mathbb{C})$ and the degenerate affine Hecke algebra, J. Algebra 209 (1998), 288-304. MR.1652134 (99h:17005)

[AMR] S. Ariki, A. Mathas and H. Rui, Cyclotomic Nazarov-Wenzl algebras, Nagoya Math. J. 182 (2006), 47-134. MR2235339 (2007d:20005)

[BG] K. Brown and K. Goodearl, Lectures on algebraic quantum groups, Birkhäuser, 2002. MR1898492(2003f:16067)

[B1] J. Brundan, Dual canonical bases and Kazhdan-Lusztig polynomials, J. Algebra 306 (2006), 17-46. MR.2271570 (2007m:05229)

[B2] J. Brundan, Symmetric functions, parabolic category $\mathcal{O}$ and the Springer fiber, Duke Math. J. 143 (2008), 41-79.

[BK1] J. Brundan and A. Kleshchev, Hecke-Clifford superalgebras, crystals of type $A_{2 \ell}^{(2)}$ and modular branching rules for $\widehat{S}_{n}$, Represent. Theory 5 (2001), 317-403. MR1870595 (2002j:17024)

[BK2] J. Brundan and A. Kleshchev, Representation theory of symmetric groups and their double covers, in: Groups, combinatorics and geometry (Durham, 2001), pp. 31-53, World Scientific, 2003. MR.1994959 (2004i:20016)

[BK3] J. Brundan and A. Kleshchev, Schur-Weyl duality for higher levels, to appear in Selecta Math.; math.RT/0605217.

[CL] R. Carter and G. Lusztig, On the modular representations of the general linear and symmetric groups, Math. Z. 136 (1974), 193-242. MR0354887 (50:7364)

[D] V. Drinfeld, Degenerate affine Hecke algebras and Yangians, Func. Anal. Appl. 20 (1986), 56-58. MR $831053(87 \mathrm{~m}: 22044)$

[E] D. Eisenbud, Commutative algebra with a view towards algebraic geometry, GTM 150, Springer, 1994. MR1322960 (97a:13001)

[ES] T. Enright and B. Shelton, Categories of highest weight modules: Applications to classical Hermitian symmetric pairs, Mem. Amer. Math. Soc. 67 (1987), no. 367. MR888703 (88f:22052)

[G] I. Grojnowski, Blocks of the cyclotomic Hecke algebra, preprint, 1999.

[I] R. Irving, Projective modules in the category $\mathcal{O}_{S}$ : Self-duality, Trans. Amer. Math. Soc. 291 (1985), 701-732. MR800259 (87i:17005)

[J] G. James, The representation theory of the symmetric groups, Lecture Notes in Mathematics, no. 682, Springer, 1978. MR 513828 (80g:20019)

[JM] G. James and A. Mathas, The Jantzen sum formula for cyclotomic $q$-Schur algebras. Trans. Amer. Math. Soc. 352 (2000), 5381-5404. MR.1665333 (2001b:16017)

[Ja] J. Jantzen, Kontravariante Formen aud induzierten Darstellungen halbeinfachen LieAlgebren, Math. Ann. 226 (1977), 53-65. MR0439902 (55:12783)

[Ju] A. Jucys, Symmetric polynomials and the center of the symmetric group ring, Report Math. Phys. 5 (1974), 107-112. MR0419576 (54:7597)

[Kh] M. Khovanov, Crossingless matchings and the cohomology of $(n, n)$ Springer varieties, Commun. Contemp. Math. 6 (2004), 561-577. MR2078414 (2005g:14090)

[K] A. Kleshchev, Linear and Projective Representations of Symmetric Groups, Cambridge University Press, Cambridge, 2005. MR2165457(2007b:20022)

[L] G. Lusztig, Cuspidal local systems and graded Hecke algebras. I, Inst. Hautes Études Sci. Publ. Math. 67 (1988), 145-202. MR972345 (90e:22029) 
[LM] S. Lyle and A. Mathas, Blocks of cyclotomic Hecke algebras, Adv. Math 216 (2007), 854-878. MR2351381

[Mac] I. Macdonald, Symmetric functions and Hall polynomials, Oxford Mathematical Monographs, second edition, OUP, 1995. MR 1354144 (96h:05207)

[MS] V. Mazorchuk and C. Stroppel, Projective-injective modules, Serre functors and symmetric algebras, J. Reine Angew. Math. 616 (2008), 131-165. MR2369489

[M] G. Murphy, The idempotents of the symmetric group and Nakayama's conjecture, J. Algebra 81 (1983), 258-265. MR696137 (84k:20007)

[P] K. Platt, Classifying the representation type of infinitesimal blocks of category $\mathcal{O}_{S}$, Ph.D. thesis, University of Georgia, 2008.

[R] O. Ruff, Centers of cyclotomic Sergeev superalgebras, preprint, 2008.

[S] C. Stroppel, Perverse sheaves on Grassmannians, Springer fibres and Khovanov homolog, to appear in Compositio Math.; math.RT/0608234.

[W] W. Wang, Vertex algebras and the class algebras of wreath products, Proc. London Math. Soc. 88 (2004), 381-404. MR2032512 (2005a:17029)

Department of Mathematics, University of Oregon, Eugene, Oregon 97403

E-mail address: brundan@uoregon.edu 